\title{
Magnetars: properties, origin and evolution
}

\author{
Sandro Mereghetti · José A. Pons · Andrew Melatos
}

Received: date / Accepted: date

\begin{abstract}
Magnetars are neutron stars in which a strong magnetic field is the main energy source. About two dozens of magnetars, plus several candidates, are currently known in our Galaxy and in the Magellanic Clouds. They appear as highly variable X-ray sources and, in some cases, also as radio and/or optical pulsars. Their spin periods (2-12 s) and spin-down rates $\left(\sim 10^{-13}-10^{-10} \mathrm{~s} \mathrm{~s}^{-1}\right)$ indicate external dipole fields of $\sim 10^{13-15} \mathrm{G}$, and there is evidence that even stronger magnetic fields are present inside the star and in non-dipolar magnetospheric components. Here we review the observed properties of the persistent emission from magnetars, discuss the main models proposed to explain the origin of their magnetic field and present recent developments in the study of their evolution and connection with other classes of neutron stars.
\end{abstract}

\section{Introduction}

Magnetars are neutron stars in which the main source of energy is provided by a strong magnetic field, instead of rotation, accretion, nuclear reactions, or cooling. While the bulk of rotation-powered (radio) pulsars have fields in the range $\mathrm{B} \sim 10^{11}-10^{13} \mathrm{G}$, the external magnetic field of magnetars is typically $10^{13}-10^{15} \mathrm{G}$ and it is likely that their internal field is even stronger. However, the distributions of field intensities for magnetars and "normal" neutron stars overlap: there is not a discriminating B threshold between these two classes. Indeed, the presence of a strong dipole field (typically estimated from the star spin period and spin-down rate) is not a sufficient (nor a necessary) condition to trigger "magnetarlike" activity. The latter is in fact mainly related to the presence of a significant toroidal component of the internal field, able to produce magnetospheric twists.

\section{S.Mereghetti}

INAF IASF-Milano, v. Bassini 15, I-20133 Milano, Italy

E-mail: sandro@iasf-milano.inaf.it

\section{J.A. Pons}

Departament de Física Aplicada, Universitat d'Alacant, Ap. Correus 99, E-03080 Alacant, Spain E-mail: jose.pons@ua.es

\section{A. Melatos}

School of Physics, University of Melbourne, Parkville, VIC 3010, Australia

E-mail: amelatos@unimelb.edu.au 
Magnetars are the most variable sources among the different classes of isolated neutron stars: their characterizing property is the emission, in the X-ray and soft $\gamma$-ray range, of powerful short bursts which often reach super-Eddington luminosities. More rarely, they also emit intermediate and giant flares, the latter involving the release of up to about $10^{46}$ erg in less than half a second. Magnetars also show pulsed X-ray emission with typical luminosity of $\sim 10^{35} \mathrm{erg} \mathrm{s}^{-1}$ in persistent sources, and ranging from $\sim 10^{32}$ to $10^{36} \mathrm{erg} \mathrm{s}^{-1}$ in transient ones. The pulsations, caused by the neutron star rotation, have periods of a few seconds which are secularly increasing on timescales from one thousand to several million years $\left(\dot{P} \sim 10^{-13}-10^{-10} \mathrm{~s} \mathrm{~s}^{-1}\right)$.

Due to this variety of phenomena, most of the sources that are now believed to be magnetars were initially classified in different ways and only later recognized as members of the same class of astrophysical objects. Bursts from magnetars had been observed since the end of the 1970s (Mazets et al. 1979a b). They were initially classified as a sub-class of $\gamma$-ray bursts, with the peculiarity of a softer spectrum and of coming repeatedly from the same sky directions (Norris et al. 1991). They were thus named soft $\gamma$-ray repeaters (SGRs). A secure identification with astrophysical objects known at other wavelengths was unfeasible with the large positional uncertainties available at that time, but their possible association with supernova remnant ${ }^{1}$ suggested a neutron star nature. Other sources that are now believed to be magnetars were discovered as persistent pulsars in the soft X-ray range $(<10$ $\mathrm{keV}$ ) and thought to be X-ray binaries powered by accretion, as most of the bright X-ray sources known at that time. It was later pointed out that their narrow period distribution, long term spin-down, soft X-ray spectrum and faint optical counterparts were at variance with the properties of pulsars in massive binaries (Mereghetti \& Stella 1995). This led to their denomination as anomalous X-ray pulsars (AXPs).

We now believe that SGRs and AXPs are a single class of objects. In fact, when the persistent X-ray counterparts of SGRs were identified (Murakami et al. 1994; Rothschild et al. 1994, Hurley et al. 1999, Woods et al. 1999b), it was found that they are pulsating sources very similar to the AXPs (Kouveliotou et al. 1998, 1999, Esposito et al. 2009, Kulkarni et al. 2003), and SGR-like bursts were detected from several sources originally classified as AXPs (Gavriil et al. 2002; Kaspi et al. 2003; Woods et al. 2005). About two dozens of AXPs/SGRs are currently known in our Galaxy (plus one in each of the Magellanic Clouds) Most of them show X-ray pulsations and have been seen to emit bursts. For extensive reviews of the AXPs/SGRs observations and of the main models proposed to explain them see Woods \& Thompson (2006), Mereghetti (2008), Mereghetti (2011a), Turolla \& Esposito (2013).

We believe that the most successful explanation of the AXPs/SGRs is provided by the magnetar model (Thompson \& Duncan 1995, 1996), according to which they are neutron stars powered by a strong magnetic field. Alternative intepretations based on isolated neutron stars accreting from fall-back disks formed after the supernova explosion (see, e.g., Alpar 2001, Trümper et al. 2010) require some additional process, besides accretion, in order to explain the powerful bursts and flares observed from these sources.

In fact, the suggestion that SGRs are neutron stars powered by magnetic energy was first proposed to interpret the exceptional propertie ${ }^{3}$ of the giant flare emitted by SGR 0526-66 on March 5, 1979 (Paczynski 1992; Duncan \& Thompson| 1992). In the following years,

\footnotetext{
1 Ironically, it is now known that the nebulae associated to two of the three first discovered SGRs are not supernova remnants.

2 An updated list is mantained at http://www.physics.mcgill.ca/ pulsar/magnetar/main.html (Olausen \& Kaspi 2014).

3 The association with the supernova remnant N49 in the Large Magellanic Cloud yielded the distance and energetics of this event.
} 
the original magnetar model has been considerably developed and expanded and it provides now the best explanation for the rich diversity of AXPs and SGRs phenomenology (see, e.g., Beloborodov 2011). One essential feature of the magnetar model is the presence of significant twists in the magnetosphere (Thompson et al. 2002; Beloborodov \& Thompson 2007), resulting in a structure quite different from that of the simple dipolar geometry assumed for normal radio pulsars and in magnetospheric currents with a charge density much larger than the classical Goldreich-Julian value. Bursts and flares can be explained by sudden releases of energy in the star interior leading to fractures in the crust (Thompson \& Duncan 1995), by field reconnection events in the magnetosphere analogous to those occurring in the Sun (Lyutikov 2006), or by pair plasma fireballs produced by discontinuities in the propagation of fast MHD waves in the magnetosphere (Heyl \& Hernquist 2005).

In Section 2 we describe the properties of the so called "persistent" emission ${ }^{4}$ of magnetars. In the two following sections we review the scenarios that have been advanced for the formation of magnetars (Section 3) and for their evolution (Section 4).

\section{Properties of the persistent emission}

The main manifestations of magnetars occur in the X-ray energy range. All known confirmed magnetars show pulsations in the soft X-ray band $(<10 \mathrm{keV})$ and many of them have also been detected in hard X-rays, up to $\sim 100-200 \mathrm{keV}$. About half of the known magnetars have repeatedly been observed at nearly constant X-ray luminosities of $\sim 10^{34}-10^{35}$ erg s${ }^{-1}$, with only moderate variability (a factor of a few) on long timescales (see, e.g., Mereghetti 2011a). Much larger variability is seen in the transient magnetars, which reach the luminosity level of the "constant" magnetars only during outbursts lasting weeks/months and spend the remaining time at a much fainter, quiescent level, $\sim 10^{32} \mathrm{erg} \mathrm{s}^{-1}$ or less (see Rea \& Esposito 2011, and references therein).

The outbursts of transient magnetars are often associated with the emission of short bursts or flares (e.g., Woods et al. 2005; Esposito et al. 2008; Mereghetti et al. 2009, Aptekar et al. 2009: van der Horst et al. 2010). Short bursts are also emitted by the "constant" magnetars, but they have never been detected from a transient magnetar in the quiescent luminosity level.

\subsection{X-ray pulsations}

The presence of regular pulsations with secularly increasing period, caused by the slowing down of the neutron star rotational velocity, is one of the distinctive properties of magnetars and provides a very useful diagnostic tool for their study. The 23 currently known magnetars have spin periods in a very narrow range (2-12 s), while their period derivatives span five orders of magnitude. Most of them have $\dot{P}$ in the range $10^{-12}-10^{-10} \mathrm{~s} \mathrm{~s}^{-1}$, but in recent years a few "low- $\dot{P}$ magnetars" have been discovered, with spin-down rates as small as $4 \times 10^{-15} \mathrm{~s} \mathrm{~s}^{-1}$, well in the range of those of rotation-powered pulsars (Rea et al. 2010 . 2012, 2013b, An et al. 2013b, Scholz et al. 2014, Rea et al.2014). The observed distribution of magnetars in the pulsar $P-\dot{P}$ diagram gives information on their evolution and relation with other classes of neutron stars. The lack of observed magnetars with periods longer

\footnotetext{
4 In the lack of a better nomenclature, we use this adjective somehow improperly also for transient and variable sources, just to distinguish this emission from that of the short bursts and of the intermediate/giant flares.
} 
than $12 \mathrm{~s}$ indicates that the spin-down mechanism becomes highly inefficient at large ages and/or that old magnetars become more difficult to detect, for example because their X-ray luminosity decreases and they emit bursts less frequently. The most obvious explanation to account for these effects is magnetic field decay (Colpi et al. 2000; Dall'Osso et al. 2012), as discussed in detail in Section 4

Magnetars display X-ray pulse profiles with a variety of shapes (from simple sinusoids to multipeaked) and spanning a large range of pulsed fractions (from less than $10 \%$ to nearly $100 \%$ ). The pulse profiles are energy-dependent (with a tendency toward more complex shapes with increasing energy) and, in many sources, time-variable. Changes in pulse profiles are often connected with bursts/flares and/or glitches, but also long term variations, apparently unrelated to particular events, have been observed. Some examples of pulse profiles are shown in Figs. 1 and 2

The spin-down of magnetars is attributed to the angular momentum carried away by (time-variable) magnetized outflows and to dipole radiation losses. In a twisted magnetosphere, the latter effect produces a higher spin-down rate than in pulsars with dipolar field because the twist inflates the poloidal lines and increases the magnetic field at the light cylinder. Variations in the spin-down rate have been detected in practically all the magnetars for which good timing data extending over long periods are available. The $\dot{P}$ variations are generally smaller than $\sim 50 \%$, but changes as large as a factor of ten over timescales of weeks have sometimes been observed (Gavriil \& Kaspi 2004, Dib et al. 2009). The variations in $\dot{P}$ observed in several magnetars are a proof of the dynamic nature of their magnetospheres. Since both the torque and the magnetospheric currents are driven by variations in the twisting of the field lines (Beloborodov|2009), some correlations between the spin-down rate and $\mathrm{X}$-ray emission properties can be expected, and indeed they have been observed (see Section 2.6).

Glitches have been observed in many magnetars (Kaspi et al. 2000, Dall'Osso et al. 2003, Woods et al. 2004; Dib et al. 2009). They involve fractional frequency changes $\Delta v / v \sim 10^{-7}-10^{-4}$, similar to those of the strongest glitches of radio pulsars (Dib et al. 2008 , but the apparent paucity of magnetars glitches with smaller $\Delta v / v$ is probably a selection effect. The relation between magnetar glitches and observed changes in the properties of their X-ray radiation (e.g. bursts, flux or pulse profile changes, etc...) is extensively discussed in Dib \& Kaspi (2014). While the majority of radiative changes are associated with glitches (or with some other timing anomaly), the converse is not true: several glitches seem to have no consequences on the emitted radiation. Two sources showed episodes in which the spin frequency apparently jumped in a short time interval to a value significantly smaller than that predicted by the observed spin-down rate. These events with negative $\Delta v$ have been called antiglitches and cannot be explained with the theories of normal glitches $(\Delta v>0)$, which are based on the fact that the neutron star interior rotates faster than the crust and acts as an angular momentum reservoir. However, due to the sparse time sampling of the available data, it is not clear if these events are really occurring on a short timescale, as normal glitches. The first possible antiglitch, with $\Delta v / v=-10^{-4}$, coincided with the August 1998 giant flare of SGR 1900+14 (Woods et al. 1999a). An analysis of the pulse phases suggests that the frequency variation could have been caused by an increase of more than a factor 100 in the spin-down rate in a few hours after the giant flare (Palmer 2002). However, an alternative possibility requiring only a factor of two increase in the spin-down rate in the three months preceding the giant flare cannot be ruled out. A more convincing case for a magnetar antiglitch, with $\Delta v / v=-3 \times 10^{-7}$ and connected with a flux increase, has been recently reported for 1E 2259+586 (Archibald et al.|2013). This prompted several interpretations, involving either a sudden event, like the collision with a solid body (Huang 
\& Geng 2014), or a rapid reconfiguration of the magnetosphere with the opening of some of the closed field lines and the emission of an enhanced particle wind (Lyutikov 2013, Tong 2014).
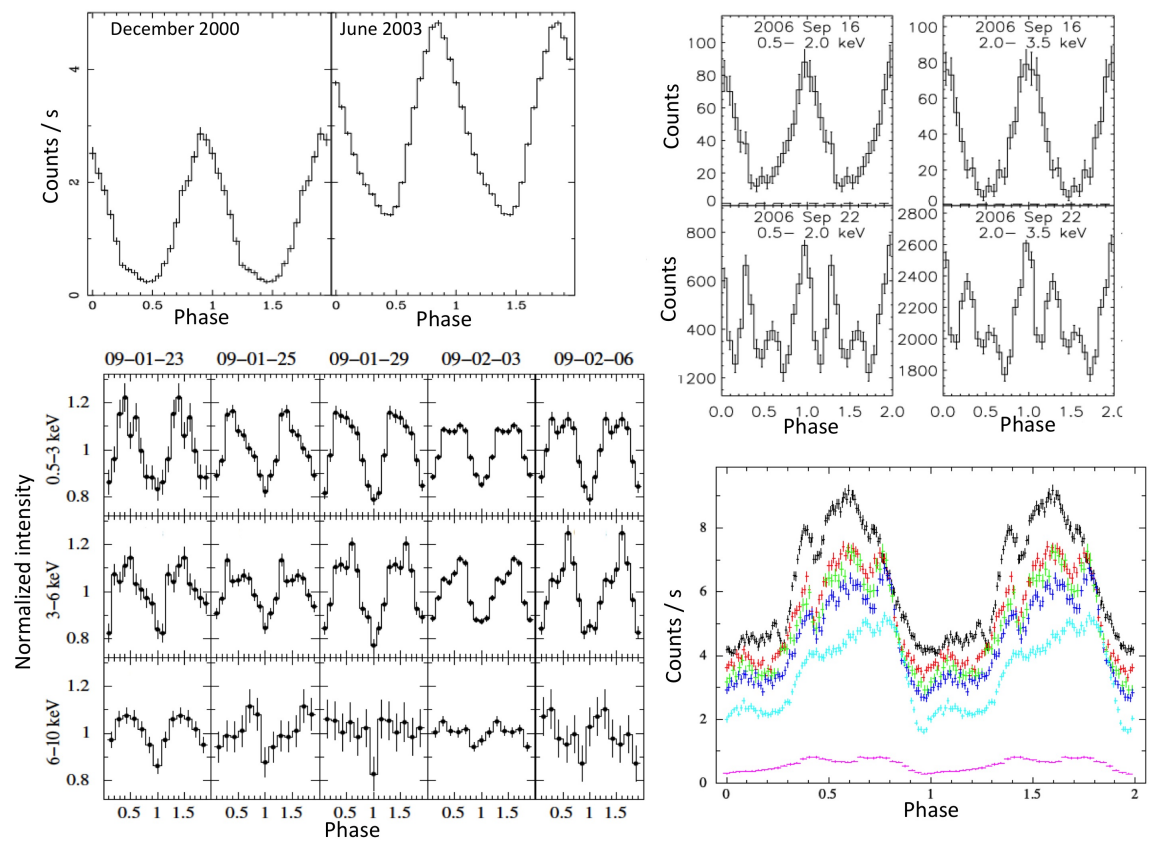

Fig. 1 Examples of X-ray pulse profiles of magnetars (for clarity, two neutron star rotations are shown in each plot). Top left panel: the pulsed fraction of the persistent magnetar 1E 1048.1-5937 anticorrelates with the luminosity (Mereghetti et al. 2004): when the flux was about twice that of the normal level, the pulsed fraction was smaller (53\% wrt $89 \%$ ); both curves refer to the $0.6-10 \mathrm{keV}$ range and were obtained with the EPIC instrument on XMM-Newton. Top right panel: pulse profile variations in two energy ranges of the transient magnetar CXOU J1647-4552 (Muno et al. 2007) in quiescence (upper panels) and during the outburst (lower panels). Bottom left panel: evolution of the pulse profiles of 1E 1547.0-5408 during the decaying phase of the January 2009 outburst (Bernardini et al. 2011). Bottom right panel: pulse profiles of the transient magnetar SGR 0501+4516 at different luminosity levels (Camero et al. 2014).

\section{$2.2 \mathrm{X}$-ray spectra}

In Fig 3 we compare representative spectra of three different classes of neutron stars: a magnetar (4U 0142+61), a rotation-powered pulsar (Geminga), and an accretion-powered binary at low luminosity (X Persei). If we limit the comparison to the $\sim 2-10 \mathrm{keV}$ range, historically the first one to be explored, the most striking difference between these sources, is the softness of the magnetar spectrum. The first measured spectra of AXPs were in fact well fit by power-laws with photon indexes $\Gamma \sim 3-4$ (Parmar et al. 1998, White et al. 1987; Sugizaki et al. 1997). This characteristic spectral softness gives an immediate diagnostics to recognize magnetar candidates among newly discovered X-ray pulsars. When data of better 


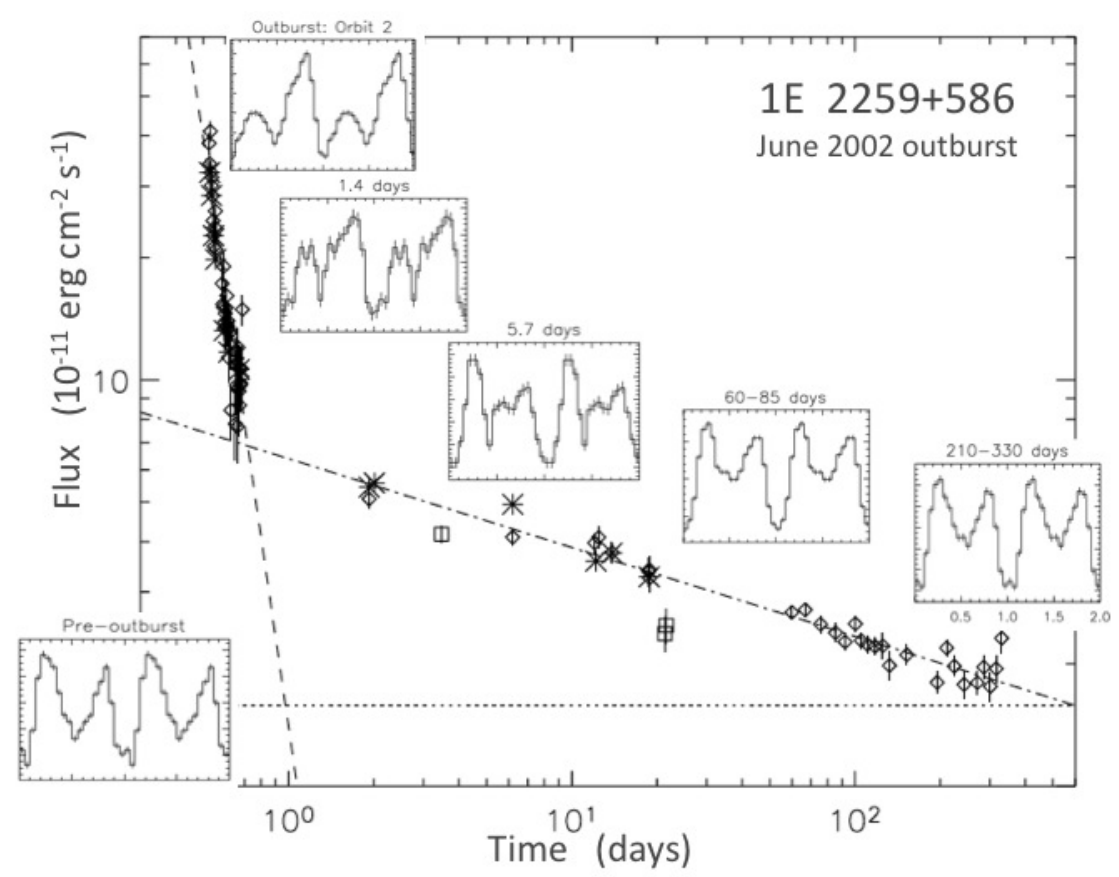

Fig. 2 Evolution of the pulse profile of 1E 2259+586 during the outburst of June 2002 (adapted from Woods et al. (2004)). The insets show pulse profiles in the $2-10 \mathrm{keV}$ obtained with the PCA instrument on RossiXTE. The inset in the lower left corner shows the pre-outburst pulse profile. Only the relative strength of the different peaks can be inferred from these profiles, which are plotted in arbitrary flux units.

quality became available, it was found that a better phenomenological fit to the magnetar spectra below $10 \mathrm{keV}$ is provided by a blackbody model with temperature $\mathrm{kT}_{B B} \sim 0.5 \mathrm{keV}$, plus either a power-law or a second blackbody component (Oosterbroek et al. 1998, White et al. 1996, Patel et al. 2001, Mereghetti et al. 2005b, 2006).

Contrary to the case of rotation-powered neutron stars, for which the thermal and nonthermal components dominate in different energy ranges (see, e.g., Geminga in Fig. 3), the higher temperature of magnetars implies that the blackbody and the power-law contribute in a similar way to the 1-10 keV flux, making more difficult to disentangle and constrain the two components. The requirement of a power-law in the soft X-ray range might simply reflect the inadequacy of a simple blackbody to fit a more complex thermal model, rather than representing a physically distinct process. For this reason, some caution is needed when drawing physical interpretations from some of the correlations between spectral parameters that have been reported in the literature.

Most magnetars are located at low Galactic latitude and thus their spectra are strongly affected by the interstellar absorption, with large column densities $\mathrm{N}_{H} \sim 10^{22}-10^{23} \mathrm{~cm}^{-2}$. The $\mathrm{N}_{H}$ values required by the blackbody plus power-law fits are often larger than those independently estimated in other ways, suggesting that the power-law cannot be extrapolated to low energy without a cut-off. Good fits are generally obtained with the sum of twoblackbody models, which can be interpreted in terms of regions with different temperatures on the star surface (Halpern \& Gotthelf 2005). Thanks to its location in the Small Magellanic 


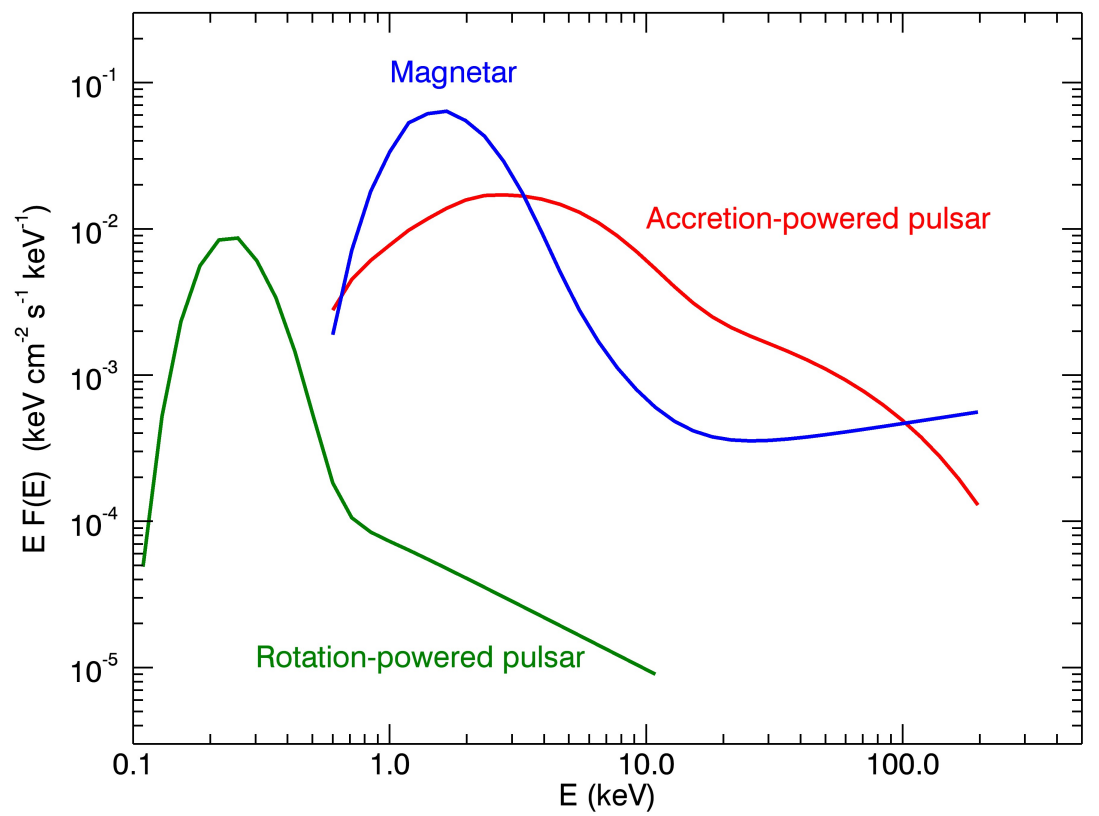

Fig. 3 Comparison of the X-ray spectra of three neutron stars representative of different classes. Red: the accretion-powered binary X Persei (adapted from Di Salvo et al.(1998)); Green: the rotation-powered pulsar Geminga (adapted from Jackson \& Halpern (2005)); Blue: the magnetically-powered AXP 4U 0142+61 (adapted from Rea et al. (2007)).

Cloud, CXOU J0100-7211 is the magnetar with the lowest interstellar absorption $\left(\mathrm{N}_{H} \sim\right.$ $6 \times 10^{20} \mathrm{~cm}^{-2}$ ) and offers the best opportunity to study the X-ray emission at low energy: its spectrum is well fit by the two-blackbody model while the power-law plus blackbody is rejected with high confidence (Tiengo et al.2008).

On the other hand, a power-law component is certainly present in the hard X-ray range. Several magnetars have been detected up to $\sim 150 \mathrm{keV}$ with large pulsed fractions and spectra flatter than those of accreting X-ray pulsars. As schematically shown in Fig. 3, the latter have exponential cut-offs at a few tens of $\mathrm{keV}$ while the spectra of magnetars extend to higher energies. The first studies of the (non-bursting) emission from AXPs/SGRs above $\sim 10 \mathrm{keV}$ were carried out with the INTEGRAL, RXTE and Suzaku satellites. Despite the limited sensitivity and imaging capabilities of the instruments operating in this range, these observations were crucial to demonstrate that the hard X-ray emission represents a nonnegligible fraction of the energy output from magnetars (Kuiper et al. 2004; Mereghetti et al. 2005a; Kuiper et al. 2006, Götz et al. 2006; den Hartog et al. 2008b a; Enoto et al. 2010c b). More sensitive observations have been obtained in the last two years with the NuSTAR satellite, thanks to the imaging capability provided by its focusing telescopes covering the 3-79 $\mathrm{keV}$ range. These observations allow to carry out spectral and variability analysis on short timescales and to spatially resolve the hard X-ray emission in crowded and/or confused regions (An et al.|2013a, Vogel et al. 2014, Kaspi et al.|2014) 
We can summarize the properties of the hard X-ray emission from magnetars as follows:

- the luminosity in the hard component is similar to that observed below $10 \mathrm{keV}$.

- fits in the range $\sim 10-200 \mathrm{keV}$ with power-law models give photon index values typically between $\Gamma \sim 1$ and 2 (except in the case of SGR 1900+14, which has $\Gamma \sim 3$, Götz et al. (2006)).

- the flux upper limits derived in the MeV region (Kuiper et al. 2006, den Hartog et al. 2006 ) imply that the spectra cannot extend as power laws to such high energies. Indeed, the data with high statistics show that curved models, like a log-parabolic function, provide better fits than simple power laws (Rea et al. 2007, den Hartog et al. 2008b a).

- the spectra of the pulsed component are harder than those of the total emission and show phase-dependent variations.

- hard X-ray emission has been observed also in transient magnetars (1E 1547.0-5408, Enoto et al. (2010a); SGR 0501+4516, Rea et al. (2009); SGR 1729-45, Mori et al. (2013); Kaspi et al. (2014)). In the case of 1E 1547.0-5408 the spectrum hardened as the flux decreased (Kuiper et al. 2012).

- no detections at higher energy have been obtained 5 The upper limits derived with Fermi in the 0.1-10 GeV range (Abdo et al. 2010, Şaşmaz Muş \& Göğüş 2010) are incompatible with earlier predictions which assumed emission from the outer magnetospheres of AXPs/SGRs (Cheng \& Zhang|2001, Zhang \& Cheng 2002). Searches for TeV emission with ground based telescopes gave negative results (Aleksić et al.|2013).

The above description of the magnetar spectra is based on simple phenomenological fits, but in recent years more physically-motivated models to interpret the observed broad band spectra have been developed (Thompson \& Beloborodov 2005, Beloborodov 2009). Two main ingredients play an important role in these models: (a) thermal emission from (a part of) the neutron star surface, which is surrounded by a thin atmosphere, and (b) the presence of a magnetosphere with a complex geometry and significant charge density. The magnetosphere affects significantly the emerging spectrum and provides additional emission components due to the presence of accelerated charges. The surface thermal emission results from interior cooling powered by magnetic field dissipation and from external heating caused by backward-flowing charges in the magnetosphere. The presence of a relatively dense plasma in the magnetospheres with a twisted configuration, a distinguishing property of magnetars, has important implications for the emitted spectrum. Resonant cyclotron scattering of the thermal photons can produce hard tails (Thompson et al. 2002).

First steps toward physical modeling taking into account the effects of the strong magnetic field on the thermal emission were done considering radiation transfer in a monodimensional approximation (Lyutikov \& Gavriil 2006, Güver et al. 2007) More realistic 3-D computations required a Monte Carlo approach to study the photon propagation in a globally twisted magnetosphere supporting the strong currents that provide a large optical depth to resonant cyclotron scattering (Fernández \& Thompson 2007; Nobili et al. 2008a). These models can successfully fit the observed spectra (Rea et al. 2008, Zane et al. 2009), but their validity above a few tens of $\mathrm{keV}$ is uncertain, because they are computed for nonrelativistic (or only mildly relativistic) particle distributions 6 . Also the assumed geometry is probably oversimplified: the seed photons may have a non-uniform and time-variable temperature distribution over the star surface and the magnetospheric twist may involve only a

\footnotetext{
5 The MeV-GeV source in the region of $1 \mathrm{E} 2259+586$ is well explained as emission from the supernova remnant CTB 109 interacting with molecular clouds (Castro et al. 2012).

6 The extension to the general case has been derived by Nobili et al. (2008b), but only limited applications have been reported (Zane et al. 2011b).
} 
limited bundle of field lines rather than being global. The modeling of phase-resolved spectra obtained during the evolution of transient magnetar outbursts is a promising approach which can give useful constrains on the models (see, e.g., Albano et al. 2010). Unfortunately, phase-resolved spectra of good statistical quality are not always available, especially in the hard X-ray range.

A model for the hard X-ray spectra of magnetars has been developed by Beloborodov (2013b) who numerically solved the radiative transfer of charged particles flowing in a large twisted magnetic loop. Relativistic electrons $\left(\gamma \sim 10^{3}\right)$ are injected by high voltage discharges close to the star surface and flow in the closed loop (rather than along open field lines as in normal pulsars). The resulting emission, strongly beamed along the loop, has a hard power-law shape below the $\mathrm{MeV}$ and is significantly suppressed at higher energy. This is in agreement with the observations, but the model parameters depend strongly on the geometry of the loop and orientation of the source (Hascoët et al. 2014). Both phase-averaged and phase-resolved spectra of magnetars recently obtained with the NuSTAR satellite in the 4$80 \mathrm{keV}$ range have been successfully fit by this model, which, however, does not yet include in a self-consistent way the low energy X-ray emission (An et al.2013a; Vogel et al.2014).
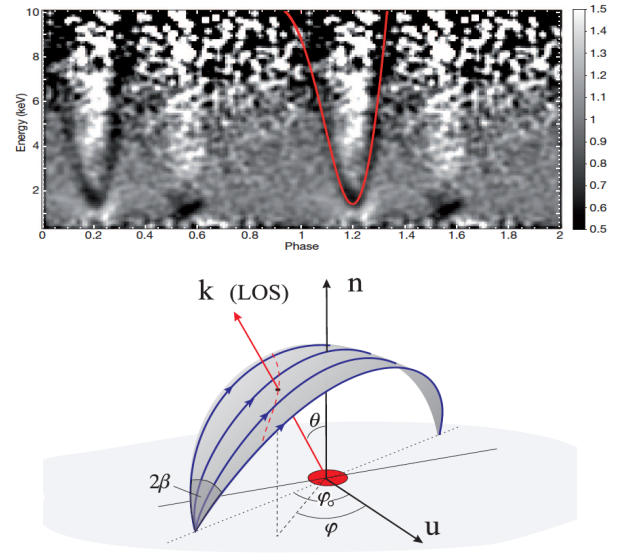

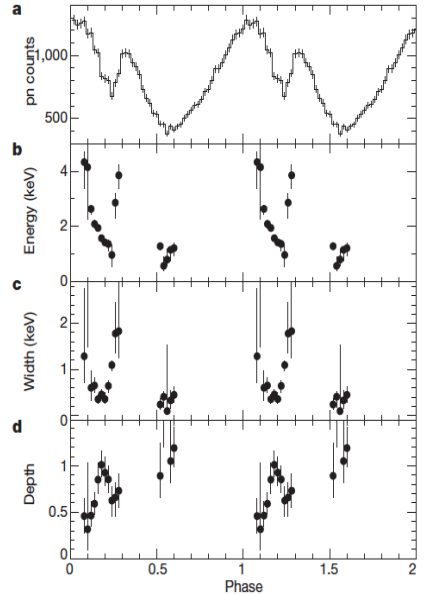

Fig. 4 Phase-dependent line in the spectrum of the transient magnetar SGR $0418+5729$ discovered with an XMM-Newton observation carried out on 2009 August 12, about two months after the beginning of the outburst (adapted from Tiengo et al. (2013)). Top left: Phase-energy image obtained by binning the source counts into 100 phase bins and 100-eV-wide energy channels and normalising to the average spectrum and light curve. The red line indicates (only on one of the two displayed cycles, for clarity) the expected phase-dependence of the line energy in the proton cyclotron model illustrated in the next panel. Bottom left: Schematic view of the model involving a magnetic loop over the X-ray emitting hot spot. The line of sight (LOS) intercepts the loop at different positions as the star rotates. The magnetic field varies along the loop, causing the observed shift in the line energy. To reproduce the observed feature, the angle between the rotation axis and the normal $\mathbf{n}$ to the surface at the spot position must be $20^{\circ}$ and the LOS must form an angle of $70^{\circ}$ with the rotation axis. Right: Results of the phase-resolved spectroscopy. From top to bottom: $0.3-10 \mathrm{keV}$ pulse profile folded at the spin period of $9.1 \mathrm{~s}(a)$; line energy $(b)$, width $(c)$, and depth $(d)$ of the cyclotron feature as a function of the spin phase. 


\subsection{Spectral features}

The detection of cyclotron lines is the most direct way to measure the magnetic field of neutron stars. This method has been successfully applied to accreting neutron stars in Xray binaries since the beginning of X-ray astronomy (see, e.g., Revnivtsev \& Mereghetti 2014). For magnetic fields of $\mathrm{B} \sim 10^{14}-10^{15} \mathrm{G}$, electron cyclotron lines are in the $\mathrm{MeV}$ energy range, where the currently available instruments are not sensitive enough to detect the magnetar emission. On the other hand, the energies of proton cyclotron lines fall in the soft X-ray range, offering, in principle, a direct way to measure the magnetic fields of these objects. This motivated extensive searches for narrow features in the persistent emission from magnetars, which however gave, until recently, only negative result: 7 Some early claims with low statistical significance (Iwasawa et al. 1992, Rea et al. 2003) could not be confirmed with more sensitive observations (Rea et al. 2005), implying either line variability or spurious detection. The best upper limits, obtained with XMM-Newton and Chandra, yield equivalent widths smaller than a few tens of eV (Tiengo et al.|2005; Juett et al.2002; Tiengo et al. 2008, Rea et al. 2009).

Line smearing, caused, e.g., by the superposition of emission from regions of different field strength, is possibly one of the effects that reduce the detectability of cyclotron lines. Phase-resolved spectroscopy could mitigate this problem, but at the cost of a worse sensitivity due to the lower counts statistics of the spectra.

The absorption line recently discovered in the transient magnetar SGR $0418+5729$ (Tiengo et al. 2013) shows indeed a strong dependence on the star rotation phase and could be discovered only thanks to the examination of phase-energy images (see Fig. 47). The line energy varies between $\sim 1$ and $\gtrsim 5 \mathrm{keV}$ within a small interval of the spin phase. This strong phase-dependence disfavours an explanation in terms of a cyclotron line from electrons, which, given the dipolar field $\mathrm{B}_{d}=6 \times 10^{12} \mathrm{G}$ inferred from the timing parameters $P=9.1$ and $\dot{P}=4 \times 10^{-15} \mathrm{~s} \mathrm{~s}^{-1}$ (Rea et al. 2013b), should be at a height of a few stellar radii. A field of $10^{14} \mathrm{G}$ at the star surface was inferred by fitting the X-ray spectrum of SGR $0418+5729$ with a magnetic atmosphere model (Güver et al.2011). As discussed in Tiengo et al. (2013), the phase-dependent absorption line is best interpreted as a cyclotron feature from protons residing in a relatively small magnetic loop with $\mathrm{B} \sim(2-10) \times 10^{14} \mathrm{G}$, much higher than $\mathrm{B}_{d}$. If this interpretation is correct, this result, besides providing a direct estimate of the magnetic field strength close to the surface of a magnetar, confirms the complex topology of the magnetosphere, in which global and/or localized twists, as predicted by the magnetar model, play an important role.

\subsection{Radio emission}

Most magnetars have not been detected in the radio band, despite being located above the death-line in the $P-\dot{P}$ diagram. The few magnetars detected in this band show radio properties very different from those of rotation-powered neutron stars. Radio pulsations were first detected in two transient magnetars: XTE J1810-197 (Camilo et al. 2006) and 1E 1547.0-5408 (Camilo et al. 2007b). They are characterized by large variability both in

\footnotetext{
7 Several features have been observed during bursts, e.g.: emission lines at $\sim 13-14 \mathrm{keV}$ in 1E 1048.1-5937 (Gavriil et al. 2002. An et al. 2014), in 4U 0142+61 (Gavriil et al. 2011), and in XTE J1810-197 (Woods et al.|2005); an absorption line at $5 \mathrm{keV}$ (and possibly its harmonics) in SGR 1806-20 (Ibrahim et al. 2003); an emission line at $6.4 \mathrm{keV}$ in SGR 1900+14 (Strohmayer \& Ibrahim 2000).
} 
flux and pulse profile shape on timescale of days, by a very flat spectrum $\left(S_{v} \propto v^{\alpha}\right.$, with $\alpha>-0.5$ ), and high polarization (Camilo et al. 2007c 2008). The first (and so far the only) magnetar discovered in the radio band, PSR J1622-4950, was reported in 2010 (Levin et al. 2010). Its radio properties are similar to those of the other radio-emitting magnetars. Although no clear signatures of magnetar-like activity have been seen in other wavelengths, its X-ray counterpart decreased in luminosity by a factor over 50 from 2007 to 2011 (Anderson et al.2012), suggesting that also in this case the radio emission is associated with a transient magnetar. The most recent addition to this small group is the transient magnetar SGR 1729-45 (Mori et al. 2013), which is particularly interesting due to its vicinity to the Galactic center. Its radio dispersion measure $\left(D M=1770 \pm 3 \mathrm{pc} \mathrm{cm}^{-2}\right)$ and Faraday rotation measure $\left(\mathrm{RM}=(-6.696 \pm 0.005) \times 10^{4} \mathrm{rad} \mathrm{m}^{-2}\right)$ are the highest among all known pulsars and indicate a distance very similar to that of the Galactic center (Eatough et al. 2013, Shannon \& Johnston 2013). At this distance, the angular separation of 3 " between SGR 1729-45 and the Galactic center black hole SgrA* corresponds to only $\sim 0.1 \mathrm{pc}$ and thus SGR 1729-45 has a non-negligible probability of being in a bound orbit with Sgr A* (Rea et al. 2013a). By comparing the DM and RM values of SGR 1729-45 with those of SgrA*, and considering the density profile of the hot gas seen in X-rays, Eatough et al. (2013) could constrain the magnetic field intensity at the beginning of the accretion flow onto the central black hole to be larger than $\sim 8 \mathrm{mG}$. If such a field is transported by the accretion flow it can be dynamically important for the accretion process on SgrA*.

The presence of radio emission gives the possibility to get very accurate positions and to measure proper motion through long baseline radio interferometry. The recently detected proper motion for SGR 1729-45 suggests that this magnetar descends from one of the massive stars in the clockwise-rotating disk around the Galactic center (Bower et al. 2015). Proper motions have been measured also for XTE J1810-197 and 1E 1547.0-5408 (Helfand et al.2007, Deller et al. 2012) in the radio band, while near IR observations yielded the proper motions of SGR 1806-20 SGR 1900+14 1E 2259+586 and 4U 0142+61 (Tendulkar et al. 2012, 2013)). These measurements correspond to transverse velocities of $\sim 100$ $300 \mathrm{~km} \mathrm{~s}^{-1}$, not dissimilar from those of rotation powered pulsars (Hobbs et al. 2005).

\subsection{Optical and infrared emission}

The study of magnetars in the optical and infrared is complicated by their intrinsic faintness at these wavelengths and by their location in strongly absorbed and crowded regions of the Galactic plane. Despite these difficulties, counterparts have been found for about one third of the known magnetars, and possible candidates have been suggested for a few other objects. The associations are certain for the three sources showing optical pulsations: 4U 0142+61 (Kern \& Martin 2002, Dhillon et al. 2005), 1E 1048.1-5937 (Dhillon et al. 2009), and SGR 0501+4516 (Dhillon et al. 2011), while the other identifications are supported by the detection of long term variability (Israel et al. 2005; Testa et al. 2008; Tam et al. 2004).

The detected counterparts have magnitudes $\sim 23-26$ in the optical band and $\mathrm{K} \sim 19-22$ in the near infrared (NIR). They are variable, but the relation between the optical and X-ray flux changes is unclear because only few truly simultaneous observations exist and different behaviors have been reported. Correlated variations were seen during the outbursts of XTE J1810-197 (Rea et al. 2004), 1E 2259+586 (Tam et al. 2004), and SGR 0501+4516 (Dhillon et al.2011), but also cases of apparently uncorrelated or anti-correlated variations were reported (Testa et al. 2008, Camilo et al. 2007a, Durant \& van Kerkwijk 2005). The 
pulse profiles of the three optically pulsed sources show a single broad peak, nearly aligned with the soft X-ray pulse, and pulsed fractions between $\sim 20 \%$ and $\sim 50 \%$.

In the context of the magnetar scenario a few ideas for the origin of the optical/NIR emission have been put forward, involving non-thermal magnetospheric emission (Eichler et al. 2002, Beloborodov \& Thompson|2007; Zane et al.,2011a, Beloborodov|2013a), but a detailed model is still lacking.

\subsection{Correlations}

Based on the small sample of seven AXPs and SGRs known at that time, Marsden \& White (2001) pointed out that the sources with the larger spin-down rate have smaller photon index in their soft X-ray spectra. The long term evolution of the power-law photon index and $\dot{P}$ in SGR 1806-20 indicates that such a correlation between spectral hardness and average spindown rate holds also for single sources (Mereghetti et al. 2005b). An updated version of the photon index versus spin-down rate plot (top left panel of Fig. 5) confirms the correlation for persistent sources (squares) and for transients in outburst (red triangles), but only for $\dot{v} \gtrsim 10^{-14} \mathrm{~s}^{-2}$ and with some exceptions. The low- $\dot{P}$ sources have spectra harder than what would be expected from the correlation seen at higher spin-down rates, which also becomes less significant if the spectra of transients in quiescence are considered (blue triangles). In Fig. 5 we show how the spectral hardness correlates with other quantities derived from the timing parameters, such as dipole field $B_{d} \propto(P \dot{P})^{1 / 2}$, charactersitic age $\tau=P / 2 \dot{P}$, and spindown power $\dot{E}_{\text {rot }} \propto \dot{P} P^{-3}$. As noted by Kaspi \& Boydstun (2010), the best correlation is that with $B_{d}$. This is often considered to support the magnetar model: a stronger, and more twisted, field causes a larger spin-down rate as well as stronger magnetospheric currents which harden the spectrum through resonant cyclotron scattering (Baring \& Harding 2007). However, the situation is probably more complicated, as also shown by the scatter of the points of Fig. 5. and other parameters might play an important role. As discussed in Section 2.2. it is difficult to disentangle the thermal and non-thermal components in the soft X-ray range ( $\lesssim 10 \mathrm{keV}$ ) and the particular geometry of the twisted bundles of magnetic field lines, not necessarily reflected in the derived $B_{d}$ values, is the most relevant factor affecting the magnetar emission properties (Beloborodov|2009).

Fig. 6 indicates that the blackbody temperatures derived from double component fits to the soft X-ray spectra of magnetars do not show significant correlations with $\dot{P}$ or $B_{d}$ (Olausen \& Kaspi 2014). However, when one compares the average temperature (or thermal luminosity) of magnetars with those of other classes of isolated neutron stars, interesting trends appear: there is a clear correlation between temperature and magnetic field Aguilera et al. 2008, Olausen et al. 2013) and the magnetars are systematically more luminous than rotation-powered neutron stars of comparable characteristic age (see Section 4 .

The first observations of persistent emission from magnetars above $\sim 20 \mathrm{keV}$ revealed a difference between the steep spectra of two SGRs with a remarkable record of bursting activity (SGR 1806-20 and SGR 1900+14) and those of the much quieter AXPs, which showed instead a significant hardening at high energy (Götz et al.2006). Enoto et al. (2010c) reported an correlation between the hardness ratio (between the $20-100 \mathrm{keV}$ and $2-10 \mathrm{keV}$ fluxes) and $B_{d}$, based on the five persistent magnetars considered by Götz et al. (2006) plus the transient SGR 0501+4516. Similar conclusions were obtained by Kaspi \& Boydstun (2010), who examined how the spectral turnover (defined as the difference between the photon indexes of soft and hard X-rays) correlates with spin-down rate and $B_{d}$. 

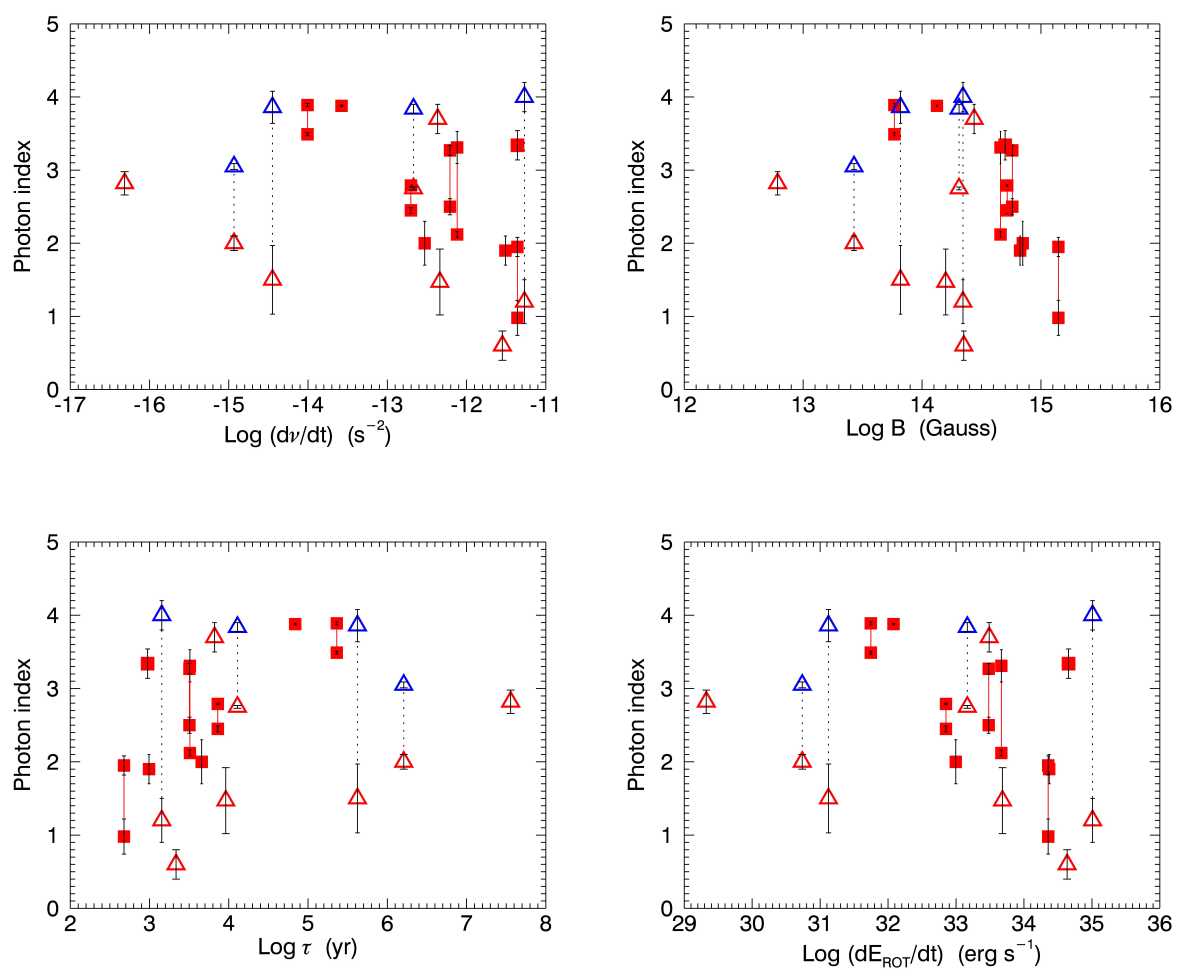

Fig. 5 Dependence of the soft X-ray power-law photon index on different parameters: $\dot{v}$ (top left panel), $\mathrm{B}_{d}$ (top right panel), $\tau$ (bottom left panel) and $\dot{E}_{\text {rot }}$ (bottom right panel). Red squares indicate persistent sources (maximum and minimum observed values are reported for several sources). Triangles indicare transient sources during outburst (red) and in during quiescence (blue).

\section{Magnetar formation and origin of magnetic field}

Scenarios of magnetar formation need to reconcile two somewhat contradictory trends. On the one hand, the strong fields of magnetars argue for special conditions at birth: a highly magnetized progenitor in the fossil field hypothesis (Woltjer 1964), or a rapidly rotating protoneutron star in the shear-driven dynamo hypothesis (Thompson \& Duncan 1993). On the other hand, the magnetar birth rate $\left(\gtrsim 0.3\right.$ century $^{-1}$; Keane \& Kramer (2008)) is comparable to rate of the core collapse supernovae $\left(1.9 \pm 1.1\right.$ century $\left.^{-1}\right)$, measured by taking a $\gamma$-ray census of radioactive ${ }^{26} \mathrm{Al}$ in the Galaxy (Diehl et al. 2006). In this respect, the birth of a magnetar is not an unusual event, especially if there is field decay (see Section 4 and Gill \& Heyl (2007)).

8 The estimate from ${ }^{26} \mathrm{Al}$ is subject to systematic uncertainties (included in the quoted error), as the isotopic yield is model dependent, and there is an unknown yield contribution from local spallation processes and specific star-forming regions. This rate is broadly consistent with extragalactic estimates (Dahlen et al. 2012 Taylor et al. 2014) which are undifferentiated by galactic type, e.g. comparative studies in the Local Volume which are limited statistically by the small number of events (Botticella et al. 2012). 

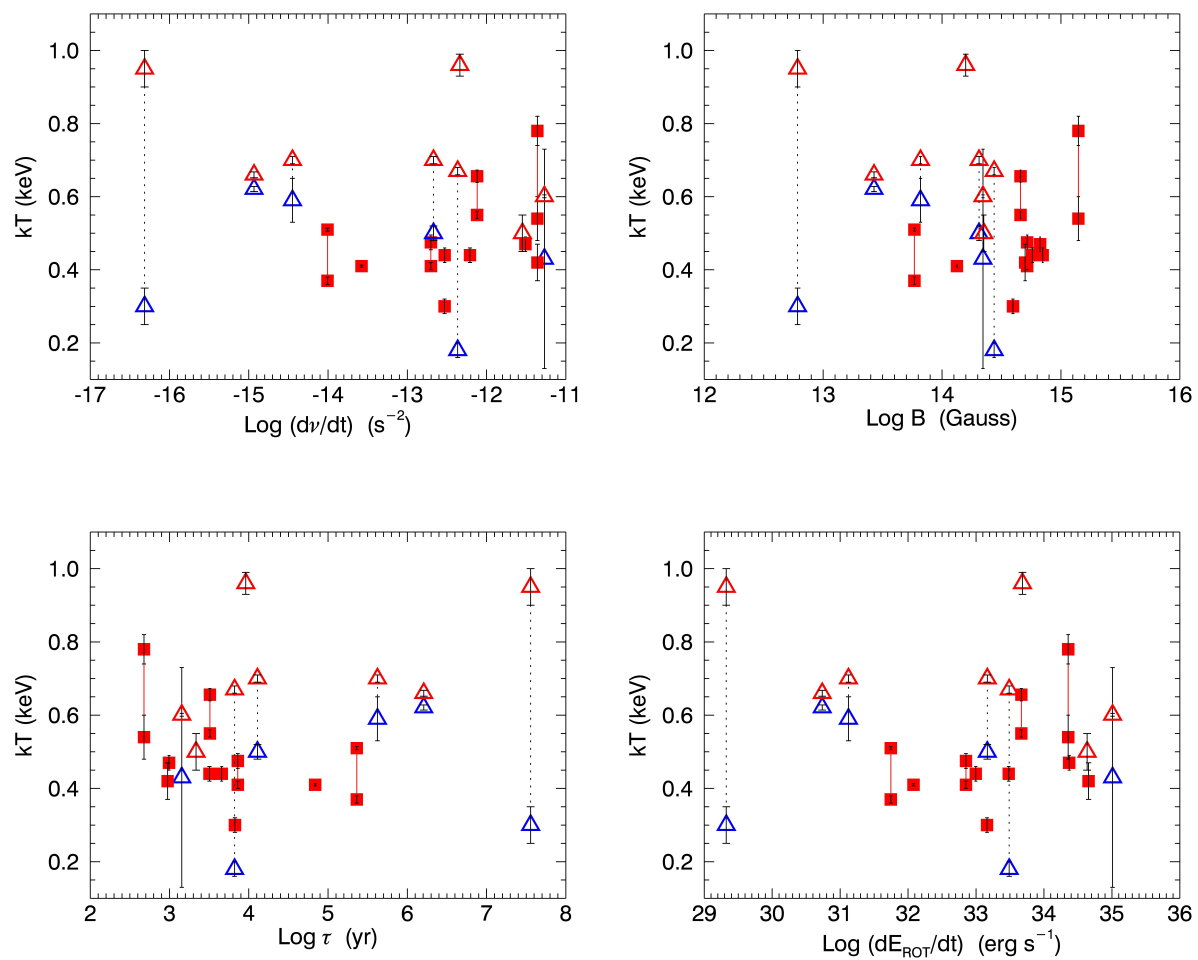

Fig. 6 Dependence of the soft X-ray blackbody temperature on different parameters: $\dot{v}$ (top left panel), $\mathrm{B}_{d}$ (top right panel), $\tau$ (bottom left panel) and $\dot{E}_{\text {rot }}$ (bottom right panel). Red squares indicate persistent sources (maximum and minimum observed values are reported for several sources). Triangles indicare transient sources during outburst (red) and in during quiescence (blue).

Current estimates of the natal magnetic field distribution of neutron stars in general, based on population synthesis studies (Faucher-Giguère \& Kaspi 2006, Kiel et al. 2008), Zeeman spectropolarimetry of progenitors (Landstreet et al. 2007; Wade et al. 2014), and gravitational wave upper limits (Dall'Osso et al. 2009; Mastrano et al. 2011; Mastrano \& Melatos 2012), are not sufficiently precise to pin down the shape of the distribution and test whether magnetars populate a second, high-field peak. In this section we present a brief summary of the advantages and disadvantages of the fossil and dynamo hypothesis for the origin of the magnetar magnetic field. The reader is referred to the paper by Ferrario et al. in this volume and to the in-depth review by Spruit (2009) for more details.

\subsection{Fossil field}

Magnetic flux conservation during the collapse of a massive progenitor, e.g. a chemically peculiar star with radius $3 \mathrm{R}_{\odot}$ and a dipole magnetic field strength $\mathrm{B}_{0} \sim 10 \mathrm{kG}$, produces a natal neutron star magnetic field nominally as large as $\mathrm{B} \sim 5 \times 10^{15} \mathrm{G}$, enough to account for all known magnetars (Woltjer 1964: Ruderman|1972). The fossil field scenario is therefore 
economical as it does not invoke a separate mechanism to produce magnetars, which should naturally derive from the strong-field tail of the progenitor distribution. Ferrario \& Wickramasinghe (2006) performed population synthesis calculations to show that the idea accounts also for the magnetization of strong-field white dwarfs, which exhibit similar mass-flux ratios. Population synthesis computations also predict that magnetars originate preferentially from the most massive $\mathrm{O}$ stars, consistent with some magnetars being associated with massive star clusters (Muno et al. 2006, Clark et al. 2014, Eikenberry et al. 2004; Vrba et al. 2000 ) and with their very small scale height on the Galactic plane (Olausen \& Kaspi 2014). Therefore magnetars should be more massive than ordinary neutron stars, a claim which cannot be tested observationally at present.

The nominal maximum field $\mathrm{B} \sim 5 \times 10^{15} \mathrm{G}$ implied by magnetic flux conservation is hard to attain for three reasons. First, only the central $\sim 2 \%$ by cross-sectional area of the progenitor collapses to form a protoneutron star, reducing B proportionally. Second, there are too few progenitors with $\mathrm{B}_{0} \gtrsim 10 \mathrm{kG}$ to account for the magnetar birth rate inferred observationally (Keane \& Kramer 2008, Woods 2008). Third, magnetic core-envelope coupling in the progenitor brakes the core too efficiently to explain the observed neutron star spin distribution (Spruit \& Phinney 1998) and leaves no room for the magnetic-dipole braking evolution normally envisaged for magnetars unless supernova kicks play a role. (Coreenvelope coupling is an issue for the dynamo scenario too.)

\subsection{Protoneutron star dynamo}

Following flux compression, the magnetic field in a protoneutron star can be amplified further (over $\sim 10 \mathrm{~s}$ ) by dynamo action driven by convection (Thompson \& Duncan 1993, Bonanno et al. 2005) or differential rotation (Braithwaite 2006; Moiseenko et al. 2006). The relatively high $(\gtrsim 10 \%)$ incidence of progenitors with $\mathrm{B}_{0} \gtrsim 0.25 \mathrm{kG}$ from spectral class $\mathrm{F} 0$ to $\mathrm{O} 4$ (Wade et al.|2014) ensures that a seed field is available.

Neutrino-driven convection leads to protoneutron star fields of about $10^{11} \mathrm{G}$ for a neutrino luminosity of $\sim 10^{44} \mathrm{erg} \mathrm{s}^{-1}$, which increases $\sim 10^{4}$-fold by flux conservation when the protoneutron star collapses to form a magnetar (Thompson \& Duncan 1993). However, these maximum field values are hard to attain, because dynamos typically operate at $\lesssim 5 \%$ of equipartition (Cook et al. 2003; Braithwaite 2006) and stratification quenches convection (Spruit 2009).

In contrast, counter-intuitively, a shear-driven dynamo operates more efficiently under stratification (Braithwaite 2006). The poloidal and toroidal field components grow in concert through the action of Tayler and/or magnetorotational instabilities (Braithwaite 2006. Moiseenko et al. 2006), as well as r-mode instabilities (Cheng \& Yu 2014); the same instabilities also prevent premature saturation caused by back-reaction stress from the wound-up toroidal field. Differential rotation can also arise from binary mergers (Wickramasinghe et al. 2014), and mean-field magnetohydrodynamics (e.g. the $\alpha$ effect and anisotropic resistivity) and superfluid circulation to assist with amplification (Mastrano et al. 2011; Melatos 2012; Gusakov \& Kantor 2013).

We do not expect to see evidence for rapid rotation at birth in the current magnetar population due to magnetic braking. However, a rapidly rotating protomagnetar is expected to power an energetic, relativistic wind for $\sim 10^{2} \mathrm{~s}$ after birth, whose energy content is deposited in the supernova remnant. X-ray observations of three supernova remnants associated with magnetars find no evidence for such "over-powering", implying initial spin periods $\gtrsim 5 \mathrm{~ms}$ (Vink \& Kuiper 2006; Martin et al.|2014), although this conclusion assumes 
an idealized Sedov expansion and neglects gravitational radiation which can nullify the overpowering issue (Dall'Osso et al. 2009).

In both dynamo scenarios, magnetic flux tends to escape buoyantly from the dynamo region (Reisenegger \& Goldreich 1992; Spruit 2009, Gusakov \& Kantor 2013). Indeed, most of the flux would be lost via this process, were it not for helicity conservation, which stabilizes the situation for linked poloidal-toroidal fields under a variety of conditions (Braithwaite \& Nordlund 2006, Lander \& Jones 2012, Akgün et al. 2013, Ciolfi \& Rezzolla 2013; Gourgouliatos \& Cumming 2014).

\section{Magneto-thermal evolution of magnetars}

In neutron stars endowed with strong magnetic fields, the temperature and magnetic field evolution are closely inter-related. On one hand, the dissipation rate of the magnetic field depends on the local value of the electrical resistivity, which is a quantity strongly dependent on temperature. On the other hand, the microphysics ingredients determining the temperature evolution (heat capacity, thermal conductivity, neutrino emission rates) are significantly modified by the presence of a strong field.

A thorough and comprehensive discussion of all the aspects involved in the magnetothermal evolution of neutron stars can be found in the recent work by Viganò et al. (2013), where the authors present results from simulations including two major novelties extending previous works (Geppert et al. 1999, Page et al. 2000, Pons \& Geppert 2007; Aguilera et al. 2008, Pons et al. 2009): the proper treatment of the important Hall term in the induction equation describing the magnetic field evolution (Viganò et al. 2012), and updated microphysics inputs (see Lai 2015, for a review on matter properties in strong fields). In this section, we briefly summarize the equations, the method, and the updated ingredients of the simulations.

\subsection{Basic equations}

For our purposes, the small structural deformations induced by rotation and magnetic field can be safely neglected. To include general relativistic effects, we consider the standard static metric

$$
d s^{2}=-c^{2} e^{2 v(r)} d t^{2}+e^{2 \lambda(r)} d r^{2}+r^{2} d \Omega^{2},
$$

where $e^{2 \lambda(r)}=1-2 G m(r) / c^{2} r, m(r)$ is the enclosed mass within radius $r$, and $v(r)$ is the metric factor accounting for redshift corrections.

The neutron star magneto-thermal evolution is described by the coupled system formed by the energy balance and the Hall induction equations. The first reads:

$$
c_{v} e^{v} \frac{\partial T}{\partial t}-\nabla \cdot\left[e^{v} \hat{\kappa} \cdot \nabla\left(e^{v} T\right)\right]=e^{2 v}\left(-\mathscr{Q}_{v}+\mathscr{Q}_{h}\right)
$$

where $c_{v}$ is the volumetric heat capacity, $\hat{\kappa}$ is the thermal conductivity tensor, $\mathscr{Q}_{v}$ are the energy losses by neutrino emission per unit volume, and $\mathscr{Q}_{h}$ is the Joule heating rate per unit volume. This is the first important coupling between the two evolution equations, because $\mathscr{Q}_{h}=\mathbf{j}^{2} / \sigma$, where $j$ is the electrical current determined by the magnetic field geometry and $\sigma$ the electrical conductivity. The second important effect of the presence of a strong magnetic field in the conduction of heat is the anisotropic conductivity tensor $(\hat{\kappa})$, and the last one is that the magnetic field also affects the rate of neutrino processes, $\mathscr{Q}_{v}$. 
In the crust, ions form a Coulomb lattice, while electrons are relativistic, degenerate and can almost freely flow, providing the currents that sustain the magnetic field. The evolution of the magnetic field is governed by the Hall induction equation which, using the same notation as in Pons et al. (2009), has the form:

$$
\frac{\partial \mathbf{B}}{\partial t}=-\nabla \times\left[\frac{c^{2}}{4 \pi \sigma} \nabla \times\left(e^{v} \mathbf{B}\right)+\frac{c}{4 \pi e n_{e}}\left[\nabla \times\left(e^{v} \mathbf{B}\right)\right] \times \mathbf{B}\right]
$$

where the conductivity $\sigma$ takes into account all the electron processes, which are strongly temperature-dependent, thus resulting in the strong coupling of the magnetic field evolution to the local evolution of temperature. The first term on the right hand side accounts for Ohmic dissipation, while the second term is the Hall term. The previous equation can be cast as

$$
\frac{\partial \mathbf{B}}{\partial t}=-\nabla \times\left\{\eta\left(\nabla \times\left(e^{v} \mathbf{B}\right)+\omega_{B} \tau_{e}\left[\nabla \times\left(e^{v} \mathbf{B}\right)\right]\right) \times \mathbf{B}\right\}
$$

where we have introduced the magnetic diffusivity $\eta=\frac{c^{2}}{4 \pi \sigma}$ and the magnetization parameter $\omega_{B} \tau_{e} \equiv \frac{\sigma B}{c e n_{e}}$ (where $\omega_{B}=e B / m_{e}^{*} c$ is the gyration frequency of electrons, with $\tau_{e}$ and $m_{e}^{*}$ are the relaxation time and effective mass of electrons). This term regulates whether the evolution is dominated by the diffusive term or by the Hall term. In the regime where $\omega_{B} \tau_{e} \gg 1$ (strong magnetic fields, $\gtrsim 10^{14} \mathrm{G}$, and temperatures $\lesssim 5 \times 10^{8} \mathrm{~K}$, see Pons \& Geppert 2007; Aguilera et al. 2008; Pons et al. 2009, Viganò et al. 2012 for more details), the Hall term dominates, and the induction equation acquires a hyperbolic character. The Ohmic and Hall timescales vary by orders of magnitude within the crust and during the evolution, depending strongly on density, temperature, and magnetic field intensity and curvature. The main effect of the Hall term is to transfer part of the magnetic energy from large to small scales, as well as between poloidal and toroidal components. In the case of strong toroidal components, it also leads to the formation of discontinuities of the tangential components of the magnetic field, i.e. current sheets, where the dissipation is strongly enhanced (Viganò et al. 2012). This directly affects the thermal evolution through the term $\mathscr{Q}_{h}$ in Eq. 22.

The previous equations provide a proper description of the physics of the crust, once the microphysical input is provided. In the neutron star core, however, the situation is more complex. The core of neutron stars (or at least a fraction of its volume) is thought to be a type II superconductor (Migdal 1959; Baym et al. 1969). The dynamics of the magnetic field in the core are not clearly understood. Standard Ohmic dissipation is irrelevant due to high conductivity, but other mechanisms such as the interplay between flux-tubes and vortices, magnetic buoyancy, or ambipolar diffusion may operate to expel magnetic flux from the core on timescales comparable to the thermal evolution timescale. The detailed study of these mechanisms is still lacking, and this explains why most previous works considered models with the field confined into the crust or used a very crude approach for the magnetic fields permeating the core (Hollerbach \& Rüdiger 2002, Pons \& Geppert 2007, Pons et al. 2009. Gourgouliatos \& Cumming 2014). Some basic issues as whether or not ambipolar diffusion plays any role at all are still under debate (Glampedakis et al. 2011).

\subsection{Results}

From detailed numerical simulations solving the above system of equations, one can in principle obtain the local temperature at each point of the neutron star surface and the integrated luminosity, to be compared to observations. But the reality is more complicated. Although 


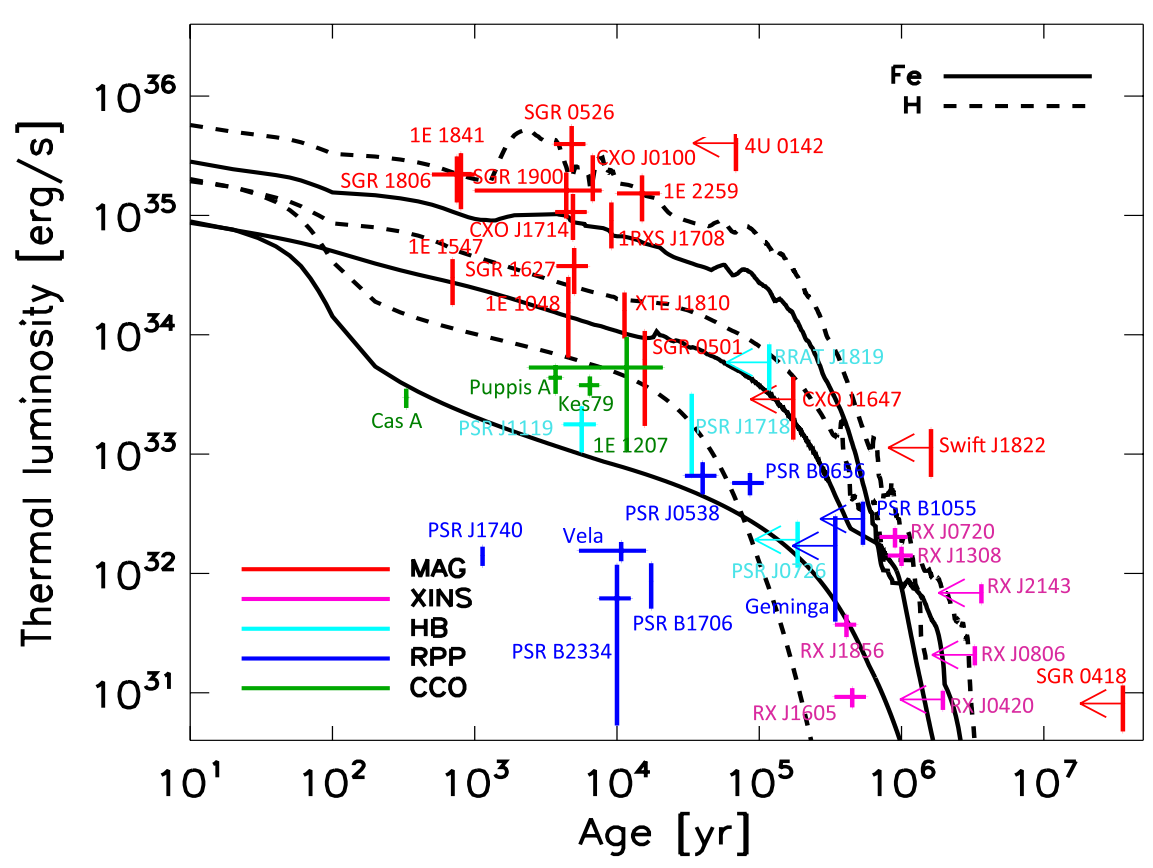

Fig. 7 Thermal X-ray luminosity versus characteristic age for magnetars (MAG) and other classes of isolated neutron stars (from Viganò et al. (2013)): X-ray dim isolated neutron stars (XINS), rotation-powered pulsars with high (HB) and normal (RPP) magnetic field, central compact objects in supernova remnants (CCO) (see, e.g., Mereghetti (2011b), for a definition of these classes of sources and their X-ray properties). The lines indicate theoretical cooling curves for different compositions of the envelope and three values of the initial magnetic field in the crust $\left(3 \times 10^{15} \mathrm{G}, 3 \times 10^{14} \mathrm{G}\right.$ and $0 \mathrm{G}$, from top to bottom). A neutron star with mass of $1.4 M_{\odot}$ and radius $11.6 \mathrm{~km}$ has been assumed.

both luminosities and temperatures can be obtained by spectral analysis, it is usually difficult to determine them accurately. The luminosity is always subject to the uncertainty in the distance estimate, while the inferred effective temperature depends on the choice of the emission model (blackbody vs. atmosphere models, composition, condensed surface, etc.), and it carries large theoretical uncertainties in the case of strong magnetic fields. It is often found that more than one model can fit equally well the data, without any clear, physically motivated preference for one of them. Photoelectric absorption from interstellar medium further constitutes a source of error in temperature measurements, since the value of the hydrogen column density is covariant with the temperature value obtained in spectral fits. Different choices for the absorption model and the metal abundances can also yield different results for the temperature. In addition, in the very common case of inhomogeneous surface temperature distributions, only an approximation with two or three regions at different temperatures is usually employed. Moreover, in the case of data with few photons and/or strong absorption features, the temperature is poorly constrained by the fit, adding a large statistical error to the systematic one.

With all the previous caveats in mind, the studies of the magneto-thermal evolution of isolated NSs, have explored the influence of their initial magnetic field strength and geometry, their mass, envelope composition, and relevant microphysical parameters such as the 


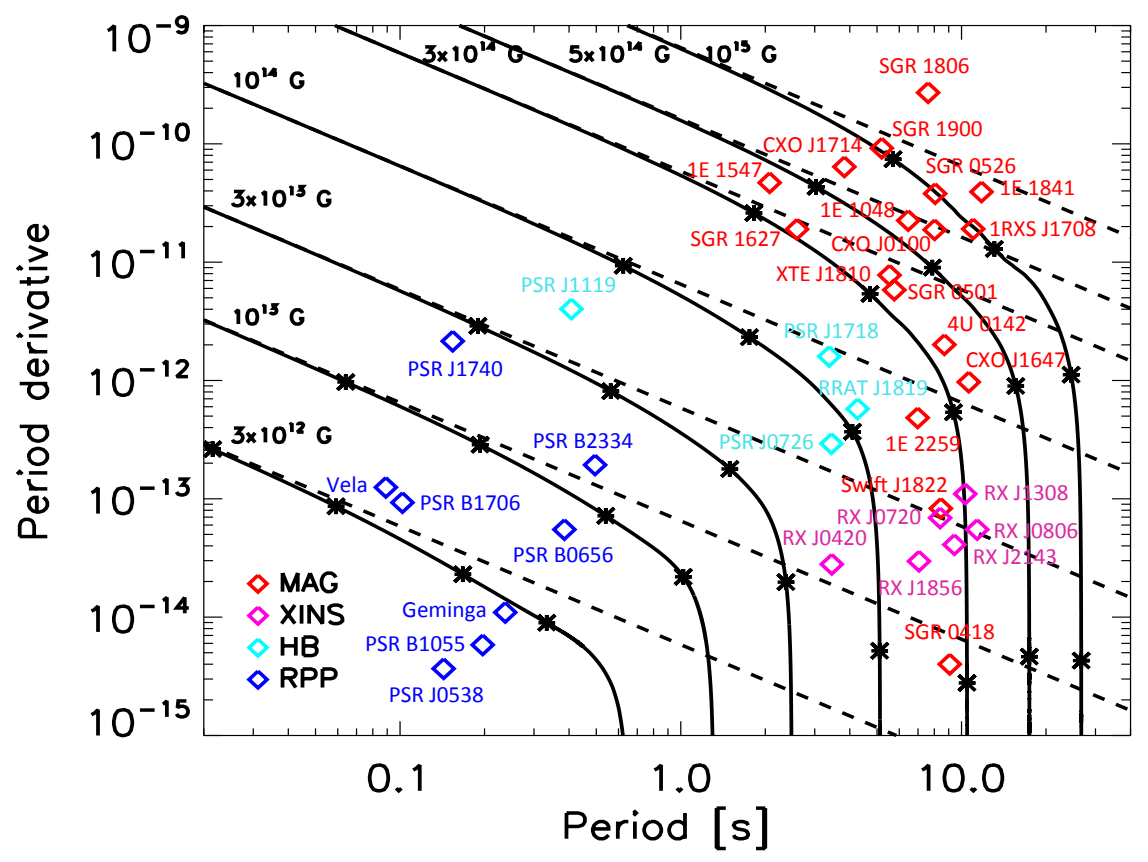

Fig. $8 P-\dot{P}$ diagram for magnetars and other classes of isolated neutron stars (from Viganò et al. (2013)). The solid lines indicate theoretical evolutionary tracks for different values of the initial magnetic field. The points corresponding to true ages of $10^{3}, 10^{4}, 10^{5}$ and $5 \times 10^{5}$ yrs are indicated by the asterisks on each lines. The dashed lines represent the evolution without magnetic field decay.

impurity content of the innermost part of the crust (the pasta region). The main findings can be summarised as follows (see Figures 7 and 8 ):

- Dependence on the magnetic field strength: The comparison between a range of theoretical models and the observations (Viganò et al.2013), has shown that, for the objects born with relatively low fields $\left(B_{p} \lesssim 10^{14} \mathrm{G}\right)$, the magnetic field has little effect on the luminosity. Sources of this group, which includes most of the "normal" radio pulsars, have luminosities which are compatible with the predictions of standard cooling models. The bulk of the magnetars, with $B_{p} \sim$ a few $\times 10^{14} \mathrm{G}$ (as estimated from their timing parameters), display luminosities generally too high to be compatible with standard cooling alone. The magneto-thermal evolutionary models with $B_{p}^{0} \sim(3-5) \times 10^{14} \mathrm{G}$ can account for their range of luminosities at the corresponding inferred ages. As these objects evolve and their magnetic fields dissipate, their observational properties (both timing and luminosities) appear compatible with those of the XDINS 9

- Relevance of the Hall term: The Hall term plays a very important role in the overall magnetic field evolution, strongly enhancing the dissipation of energy over the first $\sim 10^{6} \mathrm{yr}$ of neutron star life, with respect to the purely resistive case. This is due to two main effects: the generation of smaller structures and currents sheets, and the gradual compression of currents and toroidal field towards the crust/core interface. Hence, the rate

\footnotetext{
9 X-ray Dim Isolated Neutron Stars, a class of relatively old, purely thermally emitting neutron stars discovered by the ROSAT satellite (see, e.g., Turolla (2009), for a review of their properties).
} 
of field dissipation strongly depends on the resistivity given by the amount of impurities in the innermost region of the crust. A highly impure or amorphous inner crust produces a significant increase in the field dissipation on timescales $\gtrsim 10^{5} \mathrm{yr}$.

- Standard vs. fast neutrino cooling scenarios: For weakly magnetized objects, low mass stars $\left(M \lesssim 1.4 M_{\odot}\right)$ are systematically brighter than high mass stars, because fast neutrino processes are only active above a certain threshold density. This separation is smeared out for highly magnetized stars, for which the overwhelming effect of the magnetic field makes it hard to distinguish if fast neutrino cooling processes are acting or not, and therefore the mass dependence of the cooling curves is much smaller.

- Effect of the envelope composition: As a robust and general trend, light-element envelopes are able to maintain a higher luminosity (up to an order of magnitude) than iron envelopes for a long period of time, $\sim 10^{4} \mathrm{yr}$, regardless of the magnetic field strength. The most luminous magnetars, with estimated field strengths $\sim 10^{15} \mathrm{G}$ from their timing parameters, are barely compatible with the $10^{15} \mathrm{G}$ cooling curve with an iron envelope. However, for the same initial magnetic field, a light envelope is able to account for the luminosity of even the brightest objects.

- Importance of the initial model: The initial magnetic field configuration plays a very important role in the observational properties of the NS. If the currents sustaining the magnetic field flow in the core, their dissipation is negliglible, comparable with models in which (most of) currents flow in the crust. In particular, the presence of an initial strong dipolar, toroidal field in the crust breaks the symmetry with respect to the equator, resulting in a hemisphere warmer than the other. If most of the initial currents are instead confined into the core, then the reduced heat deposition in the crust results in a much cooler surface compared to the case in which the $B$ field lives in the crust only.

- Influence in the magnetar outburst rate: The estimated outburst rate, resulting from breaking of the crust by the strong magnetic stresses, is found to be an increasing function of the initial magnetic field strength and a decreasing function of age (Perna \& Pons 2011; Pons \& Perna 2011). A more quantitative comparison between the simulations and the observations is still not possible, due to the lack of sufficient statistics in the data.

\section{Conclusions}

Although the currently known magnetars represent only a small fraction of the observed neutron star population, they are attracting increasing interest, both from the observational and theoretical point of view. This is certainly due to their striking variability properties, diversity of multiwavelength behavior and extreme physical conditions. In the last decades, they evolved from the status of poorly understood, exotic high-energy sources to become recognized as an important class of isolated neutron stars. Although their general properties are well explained in the context of the magnetar scenario, many aspects are still poorly understood and often the observational data are not sufficient to constrain the model parameters. The transient nature of most of these sources implies that we have now discovered only a small fraction of the magnetar population. The presence of wide field of view instruments constantly monitoring the variable $\mathrm{X} / \gamma$-ray sky is extremely important to further progress in this field exploiting the improved capabilities of future observational facilities.

Acknowledgements We thank all the staff of the International Space Science Institute and the organizers of the stimulating Workshop "The Strongest Magnetic Fields in the Universe". The work of SM has been 
partially supported through the agreement ASI-INAF I/037/12/0. JAP acknowledges support of the Spanish national grant AYA 2013-42184-P and of the New Compstar COST action MP1304. AM acknowledges support of an Australian Research Council Discovery Project grant and is grateful to Nicole Darman for assistance with typesetting.

\section{References}

Abdo, A. A., Ackermann, M., Ajello, M., et al. 2010, Astrophys. J. Lett., 725, L73 Aguilera, D. N., Pons, J. A., \& Miralles, J. A. 2008, Astron. Astrophys., 486, 255 Akgün, T., Reisenegger, A., Mastrano, A., \& Marchant, P. 2013, MNRAS, 433, 2445 Albano, A., Turolla, R., Israel, G. L., et al. 2010, Astrophys. J., 722, 788

Aleksić, J., Antonelli, L. A., Antoranz, P., et al. 2013, Astron. Astrophys., 549, A23 Alpar, M. A. 2001, Astrophys. J., 554, 1245

An, H., Hascoët, R., Kaspi, V. M., et al. 2013a, Astrophys. J., 779, 163

An, H., Kaspi, V. M., Archibald, R., \& Cumming, A. 2013b, Astrophys. J., 763, 82

An, H., Kaspi, V. M., Beloborodov, A. M., et al. 2014, Astrophys. J., 790, 60

Anderson, G. E., Gaensler, B. M., Slane, P. O., et al. 2012, Astrophys. J., 751, 53

Aptekar, R. L., Cline, T. L., Frederiks, D. D., et al. 2009, Astrophys. J. Lett., 698, L82

Archibald, R. F., Kaspi, V. M., Ng, C.-Y., et al. 2013, Nature, 497, 591

Baring, M. G. \& Harding, A. K. 2007, Astrophysics and Space Science, 308, 109

Baym, G., Pethick, C., Pines, D., \& Ruderman, M. 1969, Nature, 224, 872

Beloborodov, A. M. 2009, Astrophys. J., 703, 1044

Beloborodov, A. M. 2011, in High-Energy Emission from Pulsars and their Systems, ed. D. F. Torres \& N. Rea, 299

Beloborodov, A. M. 2013a, Astrophys. J., 777, 114

Beloborodov, A. M. 2013b, Astrophys. J., 762, 13

Beloborodov, A. M. \& Thompson, C. 2007, Astrophys. J., 657, 967

Bernardini, F., Israel, G. L., Stella, L., et al. 2011, Astron. Astrophys., 529, A19

Bonanno, A., Urpin, V., \& Belvedere, G. 2005, Astron. Astrophys., 440, 199

Botticella, M. T., Smartt, S. J., Kennicutt, R. C., et al. 2012, Astron. Astrophys., 537, A132

Bower, G. C., Deller, A., Demorest, P., et al. 2015, Astrophys. J., 798, 120

Braithwaite, J. 2006, Astron. Astrophys., 449, 451

Braithwaite, J. \& Nordlund, Å. 2006, Astron. Astrophys., 450, 1077

Şaşmaz Muş, S. \& Göğüş, E. 2010, Astrophys. J., 723, 100

Camero, A., Papitto, A., Rea, N., et al. 2014, MNRAS, 438, 3291

Camilo, F., Cognard, I., Ransom, S. M., et al. 2007a, Astrophys. J., 663, 497

Camilo, F., Ransom, S. M., Halpern, J. P., \& Reynolds, J. 2007b, Astrophys. J. Lett., 666, L93

Camilo, F., Ransom, S. M., Halpern, J. P., et al. 2006, Nature, 442, 892

Camilo, F., Reynolds, J., Johnston, S., Halpern, J. P., \& Ransom, S. M. 2008, Astrophys. J., 679, 681

Camilo, F., Reynolds, J., Johnston, S., et al. 2007c, Astrophys. J. Lett., 659, L37

Castro, D., Slane, P., Ellison, D. C., \& Patnaude, D. J. 2012, Astrophys. J., 756, 88

Cheng, K. S. \& Zhang, L. 2001, Astrophys. J., 562, 918

Cheng, Q. \& Yu, Y.-W. 2014, Astrophys. J. Lett., 786, L13

Ciolfi, R. \& Rezzolla, L. 2013, MNRAS, 435, L43

Clark, J. S., Ritchie, B. W., Najarro, F., Langer, N., \& Negueruela, I. 2014, Astron. Astrophys., 565, A90

Colpi, M., Geppert, U., \& Page, D. 2000, Astrophys. J. Lett., 529, L29

Cook, J. N., Shapiro, S. L., \& Stephens, B. C. 2003, Astrophys. J., 599, 1272

Dahlen, T., Strolger, L.-G., Riess, A. G., et al. 2012, Astrophys. J., 757, 70

Dall'Osso, S., Granot, J., \& Piran, T. 2012, MNRAS, 422, 2878

Dall'Osso, S., Israel, G. L., Stella, L., Possenti, A., \& Perozzi, E. 2003, Astrophys. J., 599, 485

Dall'Osso, S., Shore, S. N., \& Stella, L. 2009, MNRAS, 398, 1869

Deller, A. T., Camilo, F., Reynolds, J. E., \& Halpern, J. P. 2012, Astrophys. J. Lett., 748, L1

den Hartog, P. R., Hermsen, W., Kuiper, L., et al. 2006, Astron. Astrophys., 451, 587

den Hartog, P. R., Kuiper, L., \& Hermsen, W. 2008a, Astron. Astrophys., 489, 263

den Hartog, P. R., Kuiper, L., Hermsen, W., et al. 2008b, Astron. Astrophys., 489, 245

Dhillon, V. S., Marsh, T. R., Hulleman, F., et al. 2005, MNRAS, 363, 609

Dhillon, V. S., Marsh, T. R., Littlefair, S. P., et al. 2011, MNRAS, 416, L16

Dhillon, V. S., Marsh, T. R., Littlefair, S. P., et al. 2009, MNRAS, 394, L112

Di Salvo, T., Burderi, L., Robba, N. R., \& Guainazzi, M. 1998, Astrophys. J., 509, 897 
Dib, R. \& Kaspi, V. M. 2014, Astrophys. J., 784, 37

Dib, R., Kaspi, V. M., \& Gavriil, F. P. 2008, Astrophys. J., 673, 1044

Dib, R., Kaspi, V. M., \& Gavriil, F. P. 2009, Astrophys. J., 702, 614

Diehl, R., Halloin, H., Kretschmer, K., et al. 2006, Nature, 439, 45

Duncan, R. C. \& Thompson, C. 1992, Astrophys. J. Lett., 392, L9

Durant, M. \& van Kerkwijk, M. H. 2005, Astrophys. J., 627, 376

Eatough, R. P., Falcke, H., Karuppusamy, R., et al. 2013, Nature, 501, 391

Eichler, D., Gedalin, M., \& Lyubarsky, Y. 2002, Astrophys. J. Lett., 578, L121

Eikenberry, S. S., Matthews, K., LaVine, J. L., et al. 2004, Astrophys. J., 616, 506

Enoto, T., Nakazawa, K., Makishima, K., et al. 2010a, PASJ, 62, 475

Enoto, T., Nakazawa, K., Makishima, K., et al. 2010b, Astrophys. J. Lett., 722, L162

Enoto, T., Rea, N., Nakagawa, Y. E., et al. 2010c, Astrophys. J., 715, 665

Esposito, P., Israel, G. L., Zane, S., et al. 2008, MNRAS, 390, L34

Esposito, P., Tiengo, A., Mereghetti, S., et al. 2009, Astrophys. J. Lett., 690, L105

Faucher-Giguère, C. A. \& Kaspi, V. M. 2006, ApJ, 643, 332

Fernández, R. \& Thompson, C. 2007, Astrophys. J., 660, 615

Ferrario, L. \& Wickramasinghe, D. 2006, MNRAS, 367, 1323

Gavriil, F. P., Dib, R., \& Kaspi, V. M. 2011, Astrophys. J., 736, 138

Gavriil, F. P. \& Kaspi, V. M. 2004, Astrophys. J. Lett., 609, L67

Gavriil, F. P., Kaspi, V. M., \& Woods, P. M. 2002, Nature, 419, 142

Geppert, U., Page, D., \& Zannias, T. 1999, Astron. Astrophys., 345, 847

Gill, R. \& Heyl, J. 2007, MNRAS, 381, 52

Glampedakis, K., Jones, D. I., \& Samuelsson, L. 2011, MNRAS, 413, 2021

Götz, D., Mereghetti, S., Tiengo, A., \& Esposito, P. 2006, Astron. Astrophys., 449, L31

Gourgouliatos, K. N. \& Cumming, A. 2014, MNRAS, 438, 1618

Gusakov, M. E. \& Kantor, E. M. 2013, Phys. Rev. D, 88, 101302

Güver, T., Göğüs, E., \& Özel, F. 2011, MNRAS, 418, 2773

Güver, T., Özel, F., Göğüss, E., \& Kouveliotou, C. 2007, Astrophys. J. Lett., 667, L73

Halpern, J. P. \& Gotthelf, E. V. 2005, Astrophys. J., 618, 874

Hascoët, R., Beloborodov, A. M., \& den Hartog, P. R. 2014, Astrophys. J. Lett., 786, L1

Helfand, D. J., Chatterjee, S., Brisken, W. F., et al. 2007, Astrophys. J., 662, 1198

Heyl, J. S. \& Hernquist, L. 2005, Astrophys. J., 618, 463

Hobbs, G., Lorimer, D. R., Lyne, A. G., \& Kramer, M. 2005, MNRAS, 360, 974

Hollerbach, R. \& Rüdiger, G. 2002, MNRAS, 337, 216

Huang, Y. F. \& Geng, J. J. 2014, Astrophys. J. Lett., 782, L20

Hurley, K., Li, P., Kouveliotou, C., et al. 1999, Astrophys. J. Lett., 510, L111

Ibrahim, A. I., Swank, J. H., \& Parke, W. 2003, Astrophys. J. Lett., 584, L17

Israel, G., Covino, S., Mignani, R., et al. 2005, Astron. Astrophys., 438, L1

Iwasawa, K., Koyama, K., \& Halpern, J. P. 1992, PASJ, 44, 9

Jackson, M. S. \& Halpern, J. P. 2005, Astrophys. J., 633, 1114

Juett, A. M., Marshall, H. L., Chakrabarty, D., \& Schulz, N. S. 2002, Astrophys. J. Lett., 568, L31

Kaspi, V. M., Archibald, R. F., Bhalerao, V., et al. 2014, Astrophys. J., 786, 84

Kaspi, V. M. \& Boydstun, K. 2010, Astrophys. J. Lett., 710, L115

Kaspi, V. M., Gavriil, F. P., Woods, P. M., et al. 2003, Astrophys. J. Lett., 588, L93

Kaspi, V. M., Lackey, J. R., \& Chakrabarty, D. 2000, Astrophys. J. Lett., 537, L31

Keane, E. F. \& Kramer, M. 2008, MNRAS, 391, 2009

Kern, B. \& Martin, C. 2002, Nature, 417, 527

Kiel, P. D., Hurley, J. R., Bailes, M., \& Murray, J. R. 2008, MNRAS, 388, 393

Kouveliotou, C., Dieters, S., Strohmayer, T., et al. 1998, Nature, 393, 235

Kouveliotou, C., Strohmayer, T., Hurley, K., et al. 1999, Astrophys. J. Lett., 510, L115

Kuiper, L., Hermsen, W., den Hartog, P. R., \& Collmar, W. 2006, Astrophys. J., 645, 556

Kuiper, L., Hermsen, W., den Hartog, P. R., \& Urama, J. O. 2012, Astrophys. J., 748, 133

Kuiper, L., Hermsen, W., \& Mendez, M. 2004, Astrophys. J., 613, 1173

Kulkarni, S. R., Kaplan, D. L., Marshall, H. L., et al. 2003, Astrophys. J., 585, 948

Lai, D. 2015, Space Science Reviews, in press

Lander, S. K. \& Jones, D. I. 2012, MNRAS, 424, 482

Landstreet, J. D., Bagnulo, S., Andretta, V., et al. 2007, Astron. Astrophys., 470, 685

Levin, L., Bailes, M., Bates, S., et al. 2010, Astrophys. J. Lett., 721, L33

Lyutikov, M. 2006, MNRAS, 367, 1594

Lyutikov, M. 2013, ArXiv e-prints 1306.2264 
Lyutikov, M. \& Gavriil, F. P. 2006, MNRAS, 368, 690

Marsden, D. \& White, N. E. 2001, Astrophys. J. Lett., 551, L155

Martin, J., Rea, N., Torres, D. F., \& Papitto, A. 2014, MNRAS, 444, 2910

Mastrano, A. \& Melatos, A. 2012, MNRAS, 421, 760

Mastrano, A., Melatos, A., Reisenegger, A., \& Akgün, T. 2011, MNRAS, 417, 2288

Mazets, E. P., Golenetskij, S. V., \& Guryan, Y. A. 1979a, Soviet Astronomy Letters, 5, 343

Mazets, E. P., Golentskii, S. V., Ilinskii, V. N., Aptekar, R. L., \& Guryan, I. A. 1979b, Nature, 282, 587

Melatos, A. 2012, Astrophys. J., 761, 32

Mereghetti, S. 2008, The Astronomy and Astrophysics Review, 15, 225

Mereghetti, S. 2011a, Advances in Space Research, 47, 1317

Mereghetti, S. 2011b, in High-Energy Emission from Pulsars and their Systems, ed. D. F. Torres \& N. Rea, 345

Mereghetti, S., Esposito, P., Tiengo, A., et al. 2006, Astrophys. J., 653, 1423

Mereghetti, S., Götz, D., Mirabel, I. F., \& Hurley, K. 2005a, Astron. Astrophys., 433, L9

Mereghetti, S., Götz, D., Weidenspointner, G., et al. 2009, Astrophys. J. Lett., 696, L74

Mereghetti, S. \& Stella, L. 1995, Astrophys. J. Lett., 442, L17

Mereghetti, S., Tiengo, A., Esposito, P., et al. 2005b, Astrophys. J., 628, 938

Mereghetti, S., Tiengo, A., Stella, L., et al. 2004, Astrophys. J., 608, 427

Migdal, A. B. 1959, Nuclear Physics, 13, 655

Moiseenko, S. G., Bisnovatyi-Kogan, G. S., \& Ardeljan, N. V. 2006, MNRAS, 370, 501

Mori, K., Gotthelf, E. V., Zhang, S., et al. 2013, Astrophys. J. Lett., 770, L23

Muno, M. P., Clark, J. S., Crowther, P. A., et al. 2006, Astrophys. J. Lett., 636, L41

Muno, M. P., Gaensler, B. M., Clark, J. S., et al. 2007, MNRAS, 378, L44

Murakami, T., Tanaka, Y., Kulkarni, S. R., et al. 1994, Nature, 368, 127

Nobili, L., Turolla, R., \& Zane, S. 2008a, MNRAS, 386, 1527

Nobili, L., Turolla, R., \& Zane, S. 2008b, MNRAS, 389, 989

Norris, J. P., Hertz, P., Wood, K. S., \& Kouveliotou, C. 1991, Astrophys. J., 366, 240

Olausen, S. A. \& Kaspi, V. M. 2014, Astrophys. J. Suppl. Ser., 212, 6

Olausen, S. A., Zhu, W. W., Vogel, J. K., et al. 2013, Astrophys. J., 764, 1

Oosterbroek, T., Parmar, A. N., Mereghetti, S., \& Israel, G. L. 1998, Astron. Astrophys., 334, 925

Paczynski, B. 1992, Acta Astronomica, 42, 145

Page, D., Geppert, U., \& Zannias, T. 2000, Astron. Astrophys., 360, 1052

Palmer, D. M. 2002, Memorie della Societa Astronomica Italiana, 73, 578

Parmar, A. N., Oosterbroek, T., Favata, F., et al. 1998, Astron. Astrophys., 330, 175

Patel, S. K., Kouveliotou, C., Woods, P. M., et al. 2001, Astrophys. J. Lett., 563, L45

Perna, R. \& Pons, J. A. 2011, Astrophys. J. Lett., 727, L51

Pons, J. A. \& Geppert, U. 2007, Astron. Astrophys., 470, 303

Pons, J. A., Miralles, J. A., \& Geppert, U. 2009, Astron. Astrophys., 496, 207

Pons, J. A. \& Perna, R. 2011, Astrophys. J., 741, 123

Rea, N. \& Esposito, P. 2011, in High-Energy Emission from Pulsars and their Systems, ed. D. F. Torres \& N. Rea, 247

Rea, N., Esposito, P., Pons, J. A., et al. 2013a, Astrophys. J. Lett., 775, L34

Rea, N., Esposito, P., Turolla, R., et al. 2010, Science, 330, 944

Rea, N., Israel, G. L., Esposito, P., et al. 2012, Astrophys. J., 754, 27

Rea, N., Israel, G. L., Pons, J. A., et al. 2013b, Astrophys. J., 770, 65

Rea, N., Israel, G. L., Stella, L., et al. 2003, Astrophys. J. Lett., 586, L65

Rea, N., Israel, G. L., Turolla, R., et al. 2009, MNRAS, 396, 2419

Rea, N., Oosterbroek, T., Zane, S., et al. 2005, MNRAS, 361, 710

Rea, N., Testa, V., Israel, G. L., et al. 2004, Astron. Astrophys., 425, L5

Rea, N., Turolla, R., Zane, S., et al. 2007, Astrophys. J. Lett., 661, L65

Rea, N., Viganò, D., Israel, G. L., Pons, J. A., \& Torres, D. F. 2014, Astrophys. J. Lett., 781, L17

Rea, N., Zane, S., Turolla, R., Lyutikov, M., \& Götz, D. 2008, Astrophys. J., 686, 1245

Reisenegger, A. \& Goldreich, P. 1992, Astrophys. J., 395, 240

Revnivtsev, M. \& Mereghetti, S. 2014, Space Science Reviews in press, arXiv:1411.5843

Rothschild, R. E., Kulkarni, S. R., \& Lingenfelter, R. E. 1994, Nature, 368, 432

Ruderman, M. 1972, ARA\&A, 10, 427

Scholz, P., Kaspi, V. M., \& Cumming, A. 2014, Astrophys. J., 786, 62

Shannon, R. M. \& Johnston, S. 2013, MNRAS, 435, L29

Spruit, H. \& Phinney, E. S. 1998, Nature, 393, 139 
Spruit, H. C. 2009, in IAU Symposium, Vol. 259, IAU Symposium, ed. K. G. Strassmeier, A. G. Kosovichev, \& J. E. Beckman, 61-74

Strohmayer, T. E. \& Ibrahim, A. I. 2000, Astrophys. J. Lett., 537, L111

Sugizaki, M., Nagase, F., Torii, K., et al. 1997, PASJ, 49, L25

Tam, C. R., Kaspi, V. M., van Kerkwijk, M. H., \& Durant, M. 2004, Astrophys. J. Lett., 617, L53

Taylor, M., Cinabro, D., Dilday, B., et al. 2014, Astrophys. J., 792, 135

Tendulkar, S. P., Cameron, P. B., \& Kulkarni, S. R. 2012, Astrophys. J., 761, 76

Tendulkar, S. P., Cameron, P. B., \& Kulkarni, S. R. 2013, Astrophys. J., 772, 31

Testa, V., Rea, N., Mignani, R. P., et al. 2008, Astron. Astrophys., 482, 607

Thompson, C. \& Beloborodov, A. M. 2005, Astrophys. J., 634, 565

Thompson, C. \& Duncan, R. C. 1993, Astrophys. J., 408, 194

Thompson, C. \& Duncan, R. C. 1995, MNRAS, 275, 255

Thompson, C. \& Duncan, R. C. 1996, Astrophys. J., 473, 322

Thompson, C., Lyutikov, M., \& Kulkarni, S. R. 2002, Astrophys. J., 574, 332

Tiengo, A., Esposito, P., \& Mereghetti, S. 2008, Astrophys. J. Lett., 680, L133

Tiengo, A., Esposito, P., Mereghetti, S., et al. 2013, Nature, 500, 312

Tiengo, A., Mereghetti, S., Turolla, R., et al. 2005, Astron. Astrophys., 437, 997

Tong, H. 2014, Astrophys. J., 784, 86

Trümper, J. E., Zezas, A., Ertan, Ü., \& Kylafis, N. D. 2010, Astron. Astrophys., 518, A46

Turolla, R. 2009, in Astrophysics and Space Science Library, Vol. 357, Astrophysics and Space Science Library, ed. W. Becker, 141

Turolla, R. \& Esposito, P. 2013, International Journal of Modern Physics D, 22, 30024

van der Horst, A. J., Connaughton, V., Kouveliotou, C., et al. 2010, Astrophys. J. Lett., 711, L1

Viganò, D., Pons, J. A., \& Miralles, J. A. 2012, Computer Physics Communications, 183, 2042

Viganò, D., Rea, N., Pons, J. A., et al. 2013, MNRAS, 434, 123

Vink, J. \& Kuiper, L. 2006, MNRAS, 370, L14

Vogel, J. K., Hascoët, R., Kaspi, V. M., et al. 2014, Astrophys. J., 789, 75

Vrba, F. J., Henden, A. A., Luginbuhl, C. B., et al. 2000, Astrophys. J. Lett., 533, L17

Wade, G. A., Grunhut, J., Alecian, E., et al. 2014, in IAU Symposium, Vol. 302, IAU Symposium, 265-269

White, N. E., Angelini, L., Ebisawa, K., Tanaka, Y., \& Ghosh, P. 1996, Astrophys. J. Lett., 463, L83

White, N. E., Mason, K. O., Giommi, P., et al. 1987, MNRAS, 226, 645

Wickramasinghe, D. T., Tout, C. A., \& Ferrario, L. 2014, MNRAS, 437, 675

Woltjer, L. 1964, Astrophys. J., 140, 1309

Woods, P. M. 2008, in American Institute of Physics Conference Series, Vol. 983, 40 Years of Pulsars: Millisecond Pulsars, Magnetars and More, ed. C. Bassa, Z. Wang, A. Cumming, \& V. M. Kaspi, 227-233

Woods, P. M., Kaspi, V. M., Thompson, C., et al. 2004, Astrophys. J., 605, 378

Woods, P. M., Kouveliotou, C., Gavriil, F. P., et al. 2005, Astrophys. J., 629, 985

Woods, P. M., Kouveliotou, C., van Paradijs, J., et al. 1999a, Astrophys. J. Lett., 524, L55

Woods, P. M., Kouveliotou, C., van Paradijs, J., et al. 1999b, Astrophys. J. Lett., 519, L139

Woods, P. M. \& Thompson, C. 2006, Soft gamma repeaters and anomalous X-ray pulsars: magnetar candidates (Compact stellar X-ray sources), 547-586

Zane, S., Nobili, L., \& Turolla, R. 2011a, in High-Energy Emission from Pulsars and their Systems, ed. D. F. Torres \& N. Rea, 329

Zane, S., Rea, N., Turolla, R., \& Nobili, L. 2009, MNRAS, 398, 1403

Zane, S., Turolla, R., Nobili, L., \& Rea, N. 2011b, Advances in Space Research, 47, 1298

Zhang, L. \& Cheng, K. S. 2002, Astrophys. J., 579, 716 